\title{
The nylon balloon for xenon loaded liquid scintillator in KamLAND-Zen 800 neutrinoless double-beta decay search experiment
}

\section{The KamLAND-Zen collaboration}

Y. Gando, ${ }^{a, *}$ A. Gando, ${ }^{a}$ T. Hachiya, ${ }^{a}$ S. Hayashida, ${ }^{a, 1}$ K. Hosokawa, ${ }^{a}$ H. Ikeda, ${ }^{a}$ T. Mitsui, $^{a}$

T. Nakada, ${ }^{a}$ S. Obara, ${ }^{e}$ H. Ozaki, ${ }^{a, f}$ J. Shirai, ${ }^{a}$ K. Ueshima, ${ }^{a, 2}$ H. Watanabe, ${ }^{a}$ S. Abe, ${ }^{a}$

K. Hata, ${ }^{a}$ A. Hayashi, ${ }^{a}$ Y. Honda, ${ }^{a}$ S. leki, ${ }^{a}$ K. Inoue, ${ }^{a, b}$ K. Ishidoshiro, ${ }^{a}$ S. Ishikawa, ${ }^{a}$

Y. Kamei, ${ }^{a}$ K. Kamizawa, ${ }^{a}$ Y. Karino, ${ }^{a}$ N. Kawada, ${ }^{a}$ T. Kinoshita, ${ }^{a}$ M. Koga, ${ }^{a}, b$ S. Matsuda, ${ }^{a}$

H. Miyake, ${ }^{a}$ K. Nakamura, ${ }^{a, b}$ K. Nemoto, ${ }^{a}$ A. Ono, ${ }^{a}$ N. Ota, ${ }^{a}$ S. Otsuka, ${ }^{a}$ Y. Shibukawa, ${ }^{a}$

I. Shimizu, ${ }^{a}$ Y. Shirahata, ${ }^{a}$ K. Soma, ${ }^{a}$ A. Suzuki, ${ }^{a}$ A.A. Suzuki, ${ }^{a}$ T. Takai, ${ }^{a}$ A. Takeuchi, ${ }^{a}$

K. Tamae, ${ }^{a}$ Y. Teraoka, ${ }^{a}$ Y. Wada, ${ }^{a}$ D. Chernyak, ${ }^{b, 3}$ A. Kozlov, ${ }^{b, 4}$ S. Yoshida, ${ }^{c}$ S. Umehara, ${ }^{d}$

Y. Takemoto, ${ }^{b, d, 5}$ K. Fushimi, ${ }^{g}$ S. Hirata, ${ }^{g}$ C. Grant, ${ }^{h}$ A. Li, ${ }^{h}$ J.G. Learned, ${ }^{i}$ J. Maricic,${ }^{i}$

B.E. Berger, ${ }^{b, j}$ B.K. Fujikawa, ${ }^{b, j}$ S. Fraker, ${ }^{k}$ A. Herman, ${ }^{k}$ E. Krupczak, ${ }^{k}$ G.L. Pease, ${ }^{k}$

L.A. Winslow, ${ }^{k}$ Y. Efremenko, ${ }^{b, l}$ H.J. Karwowski, ${ }^{m, n}$ D.M. Markoff, ${ }^{m, n}$ W. Tornow, ${ }^{m, n}$

T. O’Donnell, ${ }^{o}$ S. Dell'Oro, ${ }^{o}$ J.A. Detwiler, ${ }^{b, p}$ S. Enomoto ${ }^{b, p}$ and M.P. Decowski ${ }^{b, q}$

${ }^{a}$ Research Center for Neutrino Science, Tohoku University,

Sendai 980-8578, Japan

${ }^{b}$ Kavli Institute for the Physics and Mathematics of the Universe (WPI),

The University of Tokyo Institutes for Advanced Study, The University of Tokyo,

Kashiwa, Chiba 277-8583, Japan

${ }^{c}$ Graduate School of Science, Osaka University,

Toyonaka, Osaka 560-0043, Japan

${ }^{d}$ Research Center for Nuclear Physics, Osaka University,

1-10 Mihogaoka, Ibaraki city, Osaka 567-0042, Japan

\footnotetext{
${ }^{1}$ Present address: Imperial College London, Department of Physics, Blackett Laboratory, London SW7 2AZ, U.K. ${ }^{2}$ Present address: National Institutes for Quantum and Radiological Science and Technology (QST), Hyogo 679-5148, Japan.

${ }^{3}$ Present address: Department of Physics and Astronomy, University of Alabama, Tuscaloosa, AL 35487, U.S.A.

4Present address: National Research Nuclear University "MEPhI" (Moscow Engineering Physics Institute), Moscow, 115409, Russia.

${ }^{5}$ Present address: Kamioka Observatory, Institute for Cosmic Ray Research, The University of Tokyo, Hida, Gifu 506-1205, Japan.

${ }^{*}$ Corresponding author
} 
${ }^{e}$ Frontier Research Institute for Interdisciplinary Sciences, Tohoku University, Sendai 980-8578, Japan

${ }^{f}$ Graduate Program on Physics for the Universe (GP-PU), Tohoku University, Sendai 980-8578, Japan

${ }^{g}$ Faculty of Integrated Arts and Science, University of Tokushima,

Tokushima, 770-8502, Japan

${ }^{h}$ Department of Physics, Boston University,

Boston, MA 02215, U.S.A.

${ }^{i}$ Department of Physics and Astronomy, University of Hawaii at Manoa,

Honolulu, HI 96822, U.S.A.

${ }^{j}$ Lawrence Berkeley National Laboratory, Berkeley, CA 94720, U.S.A.

${ }^{k}$ Massachusetts Institute of Technology,

Cambridge, MA 02139, U.S.A.

${ }^{l}$ Department of Physics and Astronomy, University of Tennessee,

Knoxville, TN 37996, U.S.A.

${ }^{m}$ Triangle Universities Nuclear Laboratory,

Durham, NC 27708, U.S.A.

${ }^{n}$ Physics Departments at Duke University, North Carolina Central University and the University of North Carolina,

Chapel Hill, NC, U.S.A.

${ }^{\circ}$ Center for Neutrino Physics, Virginia Polytechnic Institute and State University,

Blacksburg, VA 24061, U.S.A.

${ }^{p}$ Center for Experimental Nuclear Physics and Astrophysics, University of Washington,

Seattle, WA 98195, U.S.A.

${ }^{q}$ Nikhef and the University of Amsterdam,

Science Park, 1098XG, Amsterdam, The Netherlands

E-mail: gando@awa.tohoku.ac.jp

AвSTRACT: The KamLAND-Zen 800 experiment is searching for the neutrinoless double-beta decay of ${ }^{136} \mathrm{Xe}$ by using ${ }^{136} \mathrm{Xe}$-loaded liquid scintillator. The liquid scintillator is enclosed inside a balloon made of thin, transparent, low-radioactivity film that we call Inner Balloon (IB). The IB, apart from guaranteeing the liquid containment, also allows to minimize the background from cosmogenic muon-spallation products and ${ }^{8} \mathrm{~B}$ solar neutrinos. Indeed these events could contribute to the total counts in the region of interest around the Q-value of the double-beta decay of ${ }^{136} \mathrm{Xe}$. In this paper, we present an overview of the IB and describe the various steps of its commissioning minimizing the radioactive contaminations, from the material selection, to the fabrication of the balloon and its installation inside the KamLAND detector. Finally, we show the impact of the IB on the KamLAND background as measured by the KamLAND detector itself.

Keywords: Double-beta decay detectors; Large detector systems for particle and astroparticle physics; Liquid detectors

ArXiv EPRINT: 2104.10452 


\section{Contents}

1 Introduction 2

2 Detector 2

2.1 KamLAND detector 2

2.2 KamLAND-Zen 800

3 Inner balloon $\quad 4$

3.1 Inner balloon design 4

3.2 Xenon-loaded liquid scintillator 4

3.3 Corrugated tube 5

4 Film selection 6

$\begin{array}{lll}\text { 4.1 Chemical compatibility and handling } & 6\end{array}$

$\begin{array}{ll}4.2 \text { Radioactive contamination } & 6\end{array}$

$\begin{array}{lll}4.3 & \text { Transparency } & 7\end{array}$

4.4 Mechanical strength 7

4.5 Tightness to xenon 8

5 Inner balloon fabrication $\quad 8$

5.1 Clean-room 8

5.2 Tool cleaning 9

5.3 Corrugated-tube cleaning 9

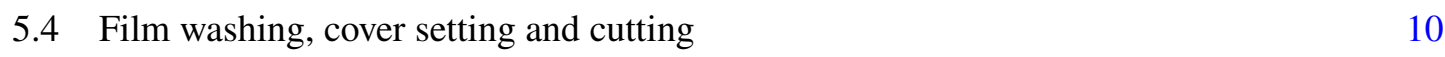

$\begin{array}{lll}5.5 \text { Welding } & 11\end{array}$

$\begin{array}{lll}5.6 & \text { Connection cylinder } & 12\end{array}$

$\begin{array}{lll}5.7 & \text { Leak check and repair } & 13\end{array}$

$\begin{array}{ll}5.8 \text { Hanging belts for the inner balloon } & 14\end{array}$

$\begin{array}{lll}5.9 & \text { Packing } & 15\end{array}$

6 Installation in the detector 16

$\begin{array}{ll}6.1 \text { Onsite preparation } & 16\end{array}$

$\begin{array}{ll}6.2 & \text { Corrugated tube connection } \\ 6.3 & \text { Sting guide }\end{array}$

$\begin{array}{lll}6.3 & \text { String guide } & 17\end{array}$

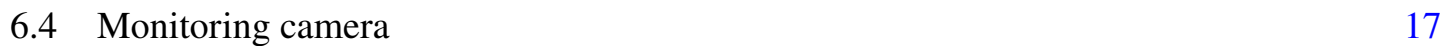

$\begin{array}{lll}6.5 & \text { Installation } & 18\end{array}$

$\begin{array}{lll}\text { 6.6 Inner balloon inflation and liquid scintillator filling } & 18\end{array}$

$\begin{array}{ll}6.7 & \text { Filling of xenon loaded liquid scintillator } \\ \end{array}$

7 Inner balloon contamination 19

$\begin{array}{llr}8 & \text { Summary } & 19\end{array}$ 


\section{Introduction}

Neutrinoless double-beta $(0 v \beta \beta)$ decay searches are direct probes of Majorana neutrinos and lepton number violation [1]. Many Grand Unified Theories predict a Majorana nature for the neutrino, and connect the neutrino's small mass to grand unification scale physics through the see-saw mechanism [2-4]. In addition, the matter-antimatter asymmetry of the universe could be explained by some leptogenesis models, which also predict Majorana neutrinos [5]. Regardless of theoretical motivations, the detection of $0 v \beta \beta$ decay would signify lepton number violation, and would mark the first observation of asymmetric matter creation in the laboratory. For these reasons, searches for $0 v \beta \beta$ decay are being vigorously pursued around the world.

KamLAND-Zen is searching for $0 v \beta \beta$ decay of the nucleus ${ }^{136} \mathrm{Xe}$. Experimentally, $0 v \beta \beta$ decay searches require a large nuclei mass and a low-background environment given the very long half-lives expected for $0 v \beta \beta$ decay ( $>10^{26} \mathrm{yr}$ ) and the $Q$-values of a few MeV. In KamLAND-Zen, the energy region surrounding the $Q$-value of the ${ }^{136} \mathrm{Xe}$ decay, $2.458 \mathrm{MeV}$ [6], is subject to environmental background from contamination present in the existing KamLAND detector material, including ${ }^{238} \mathrm{U}$ series and ${ }^{232} \mathrm{Th}$-series decays. To minimize such contamination, we developed a low-background nylon balloon to contain xenon-loaded liquid scintillator (Xe-LS) for the KamLAND-Zen experiment. The first phase, KamLAND-Zen 400 using a clean nylon balloon and enriched xenon up to $383 \mathrm{~kg}$ started in 2011 and obtained a lower limit for the $0 v \beta \beta$ decay half-life of $T_{1 / 2}^{0 v}>1.07 \times 10^{26}$ yr at $90 \%$ C.L. [7]. The next phase of KamLAND-Zen, KamLAND-Zen 800 with a cleaner balloon which was made with improved methods and new techniques started in 2018. In this paper, we describe the design of the balloon (section 3), the component materials (section 4), the production methods and clean environment procedures (section 5), the installation of the balloon into the KamLAND detector (section 6), and finally the contamination analysis of the balloon (section 7).

\section{Detector}

\subsection{KamLAND detector}

KamLAND (Kamioka Liquid scintillator Anti-Neutrino Detector) is a neutrino detector based on 1,000 tons of purified liquid-scintillator (LS) in a low background environment with 2,700 m.w.e. rock overburden in the Kamioka mine in Japan [8]. The collaboration has published measurements of reactor anti-neutrinos [9], geological anti-neutrinos [10, 11], and solar neutrinos [12, 13]. Figure 1 shows a schematic view of the KamLAND detector. It consists of a spherical stainless-steel tank, photomultiplier tubes (PMTs), buffer oil, and a 13-m-diameter balloon (outer balloon, OB) filled with LS (KamLAND LS). The KamLAND-Zen experiment introduces a second smaller balloon (inner balloon, IB) of LS loaded with ${ }^{136} \mathrm{Xe}$-enriched xenon at the center of the detector.

The outer balloon is made of five-layered nylon/EVOH film and is $135 \mu \mathrm{m}$ thick. The buffer oil between the outer balloon and the stainless-steel vessel shields the LS against external radiation. Isotropic emission photons from ionizing radiation in the LS are detected by an array of 1,325 17-inch PMTs and 554 20-inch PMTs covering 34\% of the inner surface of the detector. The vertex position and energy of the physics events are reconstructed from the timing correlation and charge of the hit PMTs, accounting for the transparency of the LS, the balloon film and buffer oil. The achieved resolution is $\sim 12 \mathrm{~cm} / \sqrt{\mathrm{E}(\mathrm{MeV})}$ for the vertex reconstruction and $\sim 6.4 \% / \sqrt{\mathrm{E}(\mathrm{MeV})}$ for 
the energy [9]. The ultra-low background $\left(\sim 10^{-17} \mathrm{~g} / \mathrm{g}\right.$ for uranium and thorium [13]) and huge volume $\left(1,200 \mathrm{~m}^{3}\right)$ of the LS make KamLAND one of the most sensitive LS detector environments for $0 v \beta \beta$ decay searches.

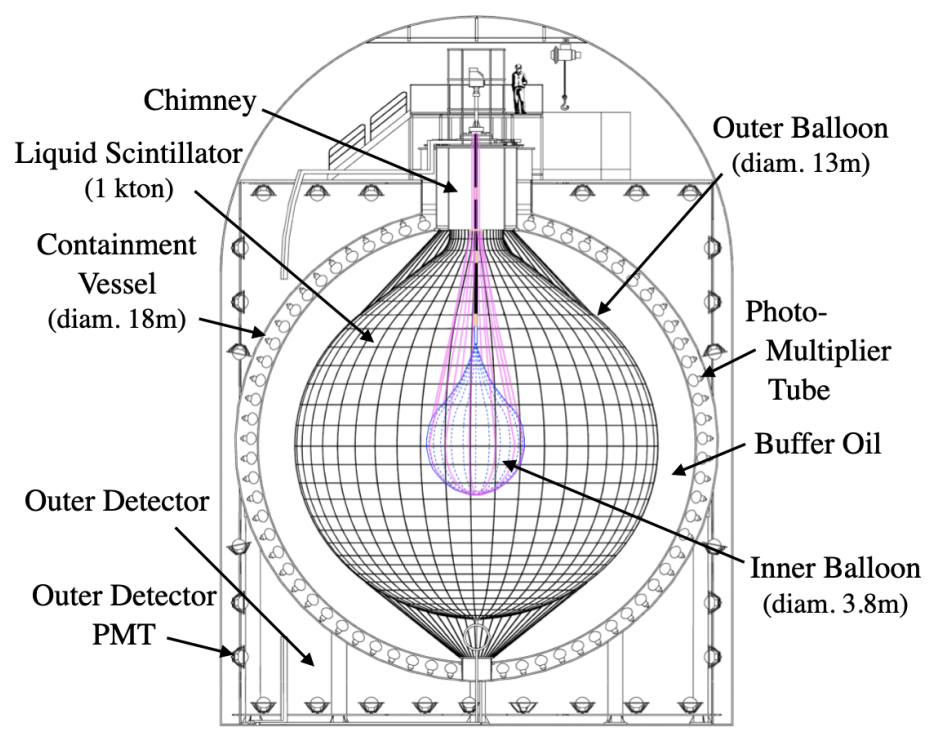

Figure 1. Schematic view of KamLAND with the IB for KamLAND-Zen 800.

\subsection{KamLAND-Zen 800}

KamLAND-Zen (KamLAND Zero neutrino double beta decay search) [14] is an experiment searching for the $0 v \beta \beta$ decay of ${ }^{136} \mathrm{Xe}$ inside KamLAND, where the IB filled with Xe-LS is suspended at the center of the detector. KamLAND-Zen 800 uses $745 \mathrm{~kg}$ xenon with a $91 \%$ isotopic abundance of ${ }^{136} \mathrm{Xe}$. Xenon is soluble in LS up to $3.1 \%$ by weight at atmospheric pressure. It can be extracted from LS by vacuum degassing and nitrogen bubbling. Backgrounds from radioactive elements in the Xe-LS are reduced by purifying the LS [13] and xenon [15] separately. The Xe-LS is transparent at its peak emission wavelength of $\sim 385 \mathrm{~nm}$.

The Q-value of ${ }^{136} \mathrm{Xe} \beta \beta$ decay is $2.458 \mathrm{MeV}$. Several backgrounds exist in this energy region: environmental radiation from ${ }^{238} \mathrm{U}$-series, pileup events from ${ }^{232} \mathrm{Th}$-series in LS, cosmogenic muon spallation products of carbon $\left({ }^{10} \mathrm{C}\right.$, etc. $)$ and xenon, and solar ${ }^{8} \mathrm{~B}$ neutrinos. The ${ }^{10} \mathrm{C}$ and pileup events can be rejected analytically: the ${ }^{10} \mathrm{C}$ is vetoed through the triple coincidence of muon, neutron, and beta/gamma-ray events from ${ }^{10} \mathrm{C}$ decay with a tagging efficiency estimated at about $90 \%$ [16]. The pileup occurs in close time coincidence within a single event window ( $\sim 200 \mathrm{~ns})$ and is also a possible background, but it can be rejected using long-time sequential decays as long as rates from other backgrounds such as ${ }^{210} \mathrm{Po}$ are low [17]. To reduce backgrounds proportional to the LS volume, such as spallation products and solar ${ }^{8} \mathrm{~B}$ neutrinos, it is advantageous to maximally load the $\beta \beta$ nuclei in the smaller volume of the inner balloon rather than using the full LS volume of KamLAND at a lower loading percentage. The balloon itself introduces radioactive backgrounds proportional to its total weight, therefore low-radioactivity materials with minimal mass are required. 
Due to its spherical shape, the access space into the detector is limited to a 50 -cm-diameter opening at the top of KamLAND. The IB is therefore installed and suspended from the chimney region at the top of the detector. To meet these mechanical constraints as well as to achieve the background requirements, we selected a balloon vessel made of thin, transparent, and foldable low-background polymeric film for the $0 v \beta \beta$ decay search in KamLAND-Zen.

\section{Inner balloon}

\subsection{Inner balloon design}

The IB is designed in a teardrop shape with smooth curves to avoid stress concentration, as shown in figure 2. We designed the IB with a volume of $31.5 \mathrm{~m}^{3}$ determined by the amount of xenon of $750 \mathrm{~kg}$ and the solubility to LS of $3.0 \mathrm{wt} \%$, which leads to the balloon radius to be $1,920 \mathrm{~mm}$. The IB consists of a main sphere formed from 24 gores, a lower polar cap, an upper cone formed from six film segments, and a 1,500-mm-long straight tube. The $25 \mu \mathrm{m}$-thick nylon film sections are connected by the heat-welding method described in section 5 . The sections are overlaid by $15 \mathrm{~mm}$ for welding at the seams.

The IB is supported by twelve 30-mm-wide nylon belts made of the same material as the IB itself. The connection of these belts to the polar harness at the bottom of the balloon is described in section 5.8. The belts are connected to twelve 0.4-mm-diameter strings (Fujinoline Corporation) made of Vectran (Kuraray Corporation) in the region of the corrugated tube, well above the IB body. The radioactive contamination of these strings is not sufficiently small for them to be employed near the IB. The strings enable easier handling of the balloon during installation. The top ends of the strings are connected to a rotary ratchet that can adjust their lengths in $1.5-\mathrm{mm}$ steps. The ratchet is suspended by a load cell (SRM200-50N, Controls Japan Company) to monitor the total weight of the IB. A string guide made of polyether ether ketone (PEEK) leads the strings into the chimney area of KamLAND. To prevent expansion or contraction due to head pressure we use a corrugated tube for the connection between the straight tube at the top of the IB and the KamLAND chimney (section 3.3).

\subsection{Xenon-loaded liquid scintillator}

To avoid damage to the IB, the density of Xe-LS is tuned to very closely match the KamLAND LS in the outer balloon. The KamLAND LS density is $0.77728 \mathrm{~kg} / \mathrm{L}$ at $15^{\circ} \mathrm{C}$. It consists of $20 \%$ pseudocumene (1,2,4-trimethylbenzene, $0.8797 \mathrm{~kg} / \mathrm{L}), 80 \%$ dodecane $\left(\mathrm{CH}_{3}\left(\mathrm{CH}_{2}\right)_{10} \mathrm{CH}_{3}, 0.7526 \mathrm{~kg} / \mathrm{L}\right)$, and $1.36 \mathrm{~g} / \mathrm{L}$ of PPO (2,5-diphenyloxazole). The density increase caused by the xenon dissolved in the LS could be counterbalanced by reducing the pseudocumene/dodecane fraction, but this would reduce the light yield, which depends on the amount of pseudocumene. We therefore replace the dodecane with decane $\left(\mathrm{CH}_{3}\left(\mathrm{CH}_{2}\right)_{8} \mathrm{CH}_{3}, 0.7339 \mathrm{~kg} / \mathrm{L}\right)$ to maximize the pseudocumene fraction in xenon-loaded LS. The decane-based LS contains $18 \%$ pseudocumene, $82 \%$ decane, and $2.38 \mathrm{~g} / \mathrm{L}$ of PPO. The density of the Xe-LS can be controlled with an accuracy of $0.02 \%$ by tuning the pseudocumene/decane fraction. The actual density of the Xe-LS is slightly heavier than the outer KamLAND LS. It is controlled within $0.1 \%$ to the KamLAND LS at all times, from installation through data acquisition, to avoid damage to the IB caused by deformation if this was to float in the KamLAND LS. The final density difference between the outer KamLAND LS and inner Xe-LS 

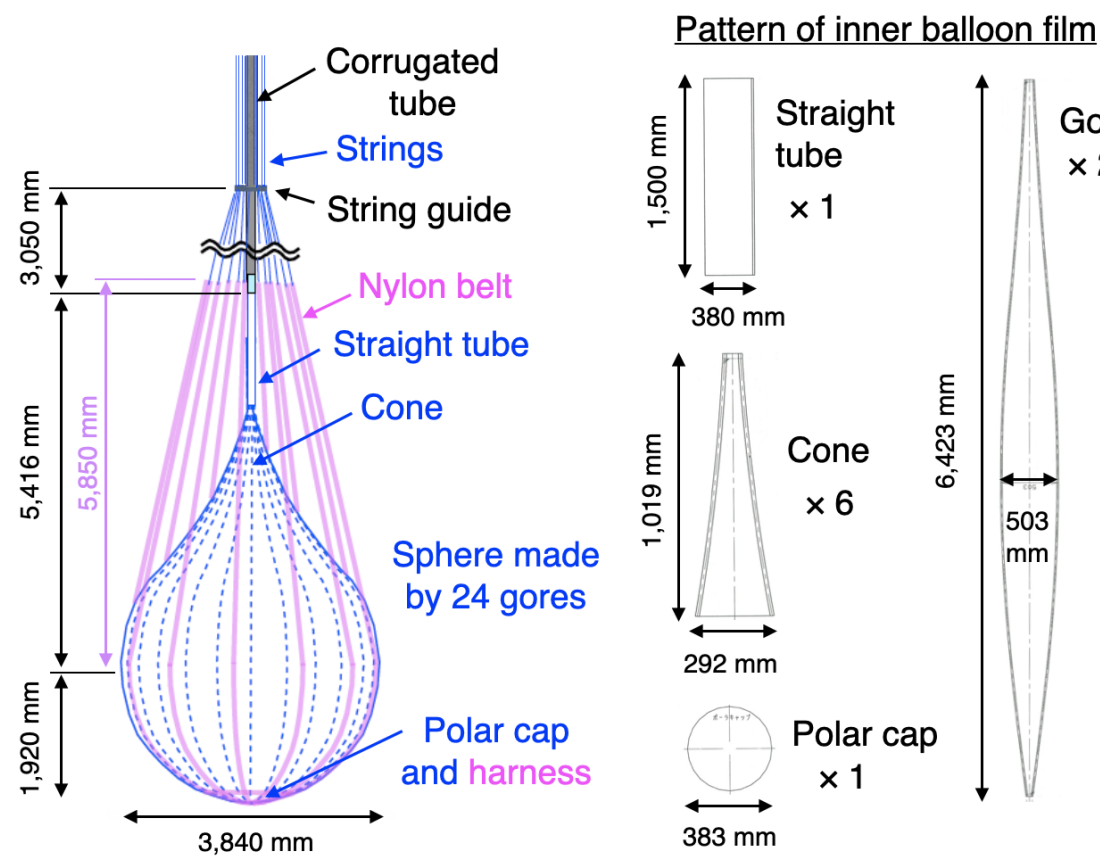

String and belt
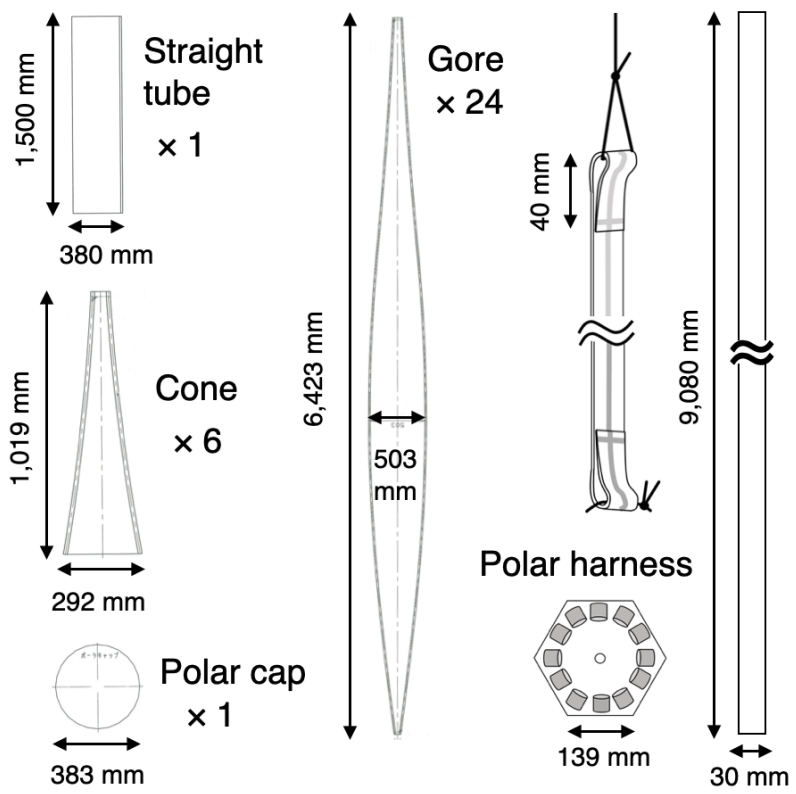

Figure 2. Schematic view of the IB, related structures, and the IB film patterns. The IB is suspended by a system of nylon belts and a harness (magenta) which support the total weight of the Xe-LS and balloon. The belts are connected to Vectran strings attached to load cells, and the weight supported by each string is monitored.

is about $0.02 \%$. The isotopic abundance of ${ }^{136} \mathrm{Xe}$ is $90.9 \%$, and the remaining components are ${ }^{124} \mathrm{Xe}-{ }^{134} \mathrm{Xe}$. The total amount of ${ }^{136} \mathrm{Xe}$ in the IB is $677 \mathrm{~kg}$.

\subsection{Corrugated tube}

The uppermost straight section of the inner vessel consists of a hard nylon tube. This design avoids contraction or expansion at the surface of LS due to the head pressure between the outer KamLAND LS and the inner Xe-LS. This tube has to be bent during the IB installation, so we used a corrugated nylon tube (CYLG-95B, PMA Company). The ${ }^{238} \mathrm{U}$ contamination of the corrugated tube was measured by ICP-MS at NTT-AT Co. to be $9 \times 10^{-12} \mathrm{~g} / \mathrm{g}$ after washing, a level acceptable for use in KamLAND. However, this contamination level is about five times larger than that of the IB film. As the tube thickness is $1 \mathrm{~mm}, 40$ times thicker than the film, the total ${ }^{238} \mathrm{U}$ contamination is 200 times that of the film, per unit surface area. To avoid $\gamma$-rays from the corrugated tube introducing backgrounds into the Xe-LS in the spherical area of the IB, the lower straight section is made of IB film for the first $1.5 \mathrm{~m}$ above the body of the IB.

The total length of corrugated tube is about $6 \mathrm{~m}$ and the diameter is about $10 \mathrm{~cm}$. It is separated into three sections to allow cleaning in the clean-room and to allow it to be kept straight during transportation. The three sections are connected with a custom-made cylinder made of PEEK as commercial connection cylinders were found not to be compatible with our LS. This connection uses fluoro-rubber O-rings, which can be used in LS. A grip piece is used to secure the connection between the cylinder and the tube and prevent it from falling out. Figure 3(a) shows the connection between the cylinder and corrugated tubes. The connection between the corrugated tube and the 
straight film tube of the IB is also via a PEEK cylinder, as shown in figure 3(b). The connection between the film tube and the rest of the IB is described in section 5.6.
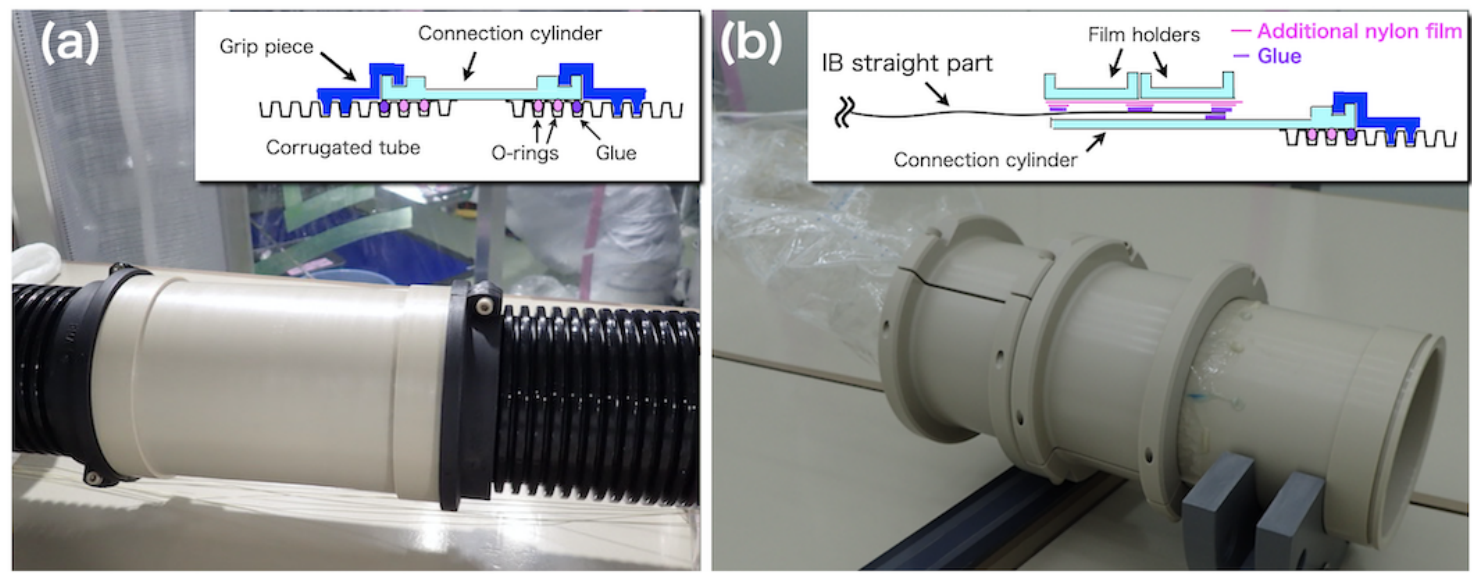

Figure 3. (a) Picture and schematic view of the connection between corrugated tubes via a connection cylinder. (b) Connection between the straight tube of the IB and the connection cylinder. The additional $25 \mu \mathrm{m}$-thick nylon film between the film holders and the IB film avoids IB damage from the film holders.

\section{Film selection}

We selected a custom-made nylon film for the IB because no commercial film satisfied our requirements on the chemical compatibility and radioactive contamination. These are described in the present section, together with the measured values.

\subsection{Chemical compatibility and handling}

Only a few kinds of film materials are compatible with a pseudocumene-based LS: ethylene tetrafluoroethylene (ETFE), nylon, and ethylene-vinylalcohol copolymer (EVOH). The outer balloon was fabricated using nylon and EVOH. However, the multi-layered film was made with glue which has radioactive impurities. We therefore had to select a single-layer film. We assessed chemical compatibility by checking for a decrease in the transparency of LS after soaking the films. For nylon and EVOH films, the transparency of the liquid samples after the film soaking was consistent within measurement error to a reference sample of the same scintillator.

EVOH has high gas impermeability. However, an EVOH film was easily torn during the production of an 80-cm-diameter test balloon. ETFE showed creep under tension, so an ETFE vessel could not stably maintain its shape. A nylon film was therefore the only candidate for the IB film.

\subsection{Radioactive contamination}

The ${ }^{214} \mathrm{Bi}$ in the ${ }^{238} \mathrm{U}$-series is one of the primary backgrounds for our $0 v \beta \beta$ decay search (figure 4(a)). The target level of ${ }^{238} \mathrm{U}$ in the balloon film is $10^{-12} \mathrm{~g} / \mathrm{g}$ for a film thickness of $25 \mu \mathrm{m}$ assuming the secular equilibrium. This level was set at $O(10 \%)$ of the estimated event rate from ${ }^{10} \mathrm{C}$ that survives a ${ }^{10} \mathrm{C}$ tagging veto. Commercial nylon films have high impurity levels $\left(\sim 10^{-9} \mathrm{~g} / \mathrm{g}\right.$ 
at ${ }^{238} \mathrm{U}$ ). However, cleaner films can be specially made without "filler". The filler is inorganic lubricant used to avoid static electricity and to prevent the film sticking to itself. We used custommade Nylon- 6 film produced by Toyobo Company from pellets produced by Ube Industries. The ${ }^{238} \mathrm{U}$ level in this nylon film was measured with ICP-MS to be $2 \times 10^{-12} \mathrm{~g} / \mathrm{g}$, sufficient for our requirements. This contamination level is consistent with that of KamLAND-Zen 400 film, and the secular equilibrium between ${ }^{238} \mathrm{U}$ and ${ }^{214} \mathrm{Bi}$ was confirmed by ${ }^{214} \mathrm{Po}(\alpha)$ energy spectrum shape analysis in KamLAND-Zen 400 [18].
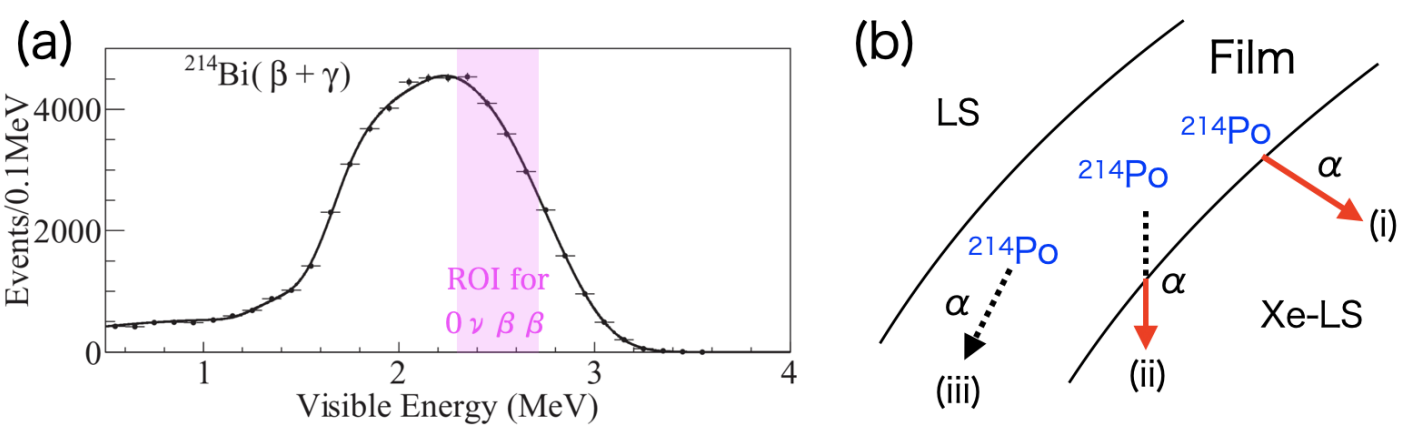

Figure 4. (a) The energy spectrum of ${ }^{214} \mathrm{Bi}(\beta+\gamma)$ with the $0 v \beta \beta$ decay region of interest (ROI) in KamLANDZen [14]. (b) Light output from $\alpha$-rays depending on the penetration route in the film. (i) Light output in Xe-LS (or LS) can be detected. (ii) The detection probability depends on the energy deposit in Xe-LS (or LS). (iii) There is no scintillation light; the event can not be detected. Reproduced with permission from [14]. (C)2012 American Physical Society.

We can $\operatorname{tag}{ }^{214} \mathrm{Bi}$ decays in the film by detecting the $\alpha$-ray from the subsequent decay of ${ }^{214} \mathrm{Po}(\tau$ $=237 \mu \mathrm{sec}$ ) under certain conditions, namely when the $\alpha$ particle leaves the film and deposits energy in the LS, producing a visible energy level above threshold (figure 4(b)). A $25 \mu \mathrm{m}$-thick film realizes $50 \%{ }^{214}$ Po detection in KamLAND. Although this ${ }^{214} \mathrm{Bi}$ rejection power is limited, this detection can be used for background estimation. Thus a $25 \mu \mathrm{m}$ thickness was preferred.

\subsection{Transparency}

A low-transparency film decreases the detector energy resolution. This leads to an increased background level by shifting the signal from two neutrino double-beta $(2 v \beta \beta)$ decays into the $0 v \beta \beta$ decay signal region. Transparency corrections could also result in a significant difference in visible energy scales inside and outside the IB, resulting in an energy distortion for events occurring near or traversing the balloon boundary. To avoid these problems, the transparency should be greater than 95\% at the effective wavelength (370-400 nm). The nylon film transparency in LS was measured to be more than $99 \%$. This level preserves the visible energy balance between energy deposited inside and outside the IB.

\subsection{Mechanical strength}

For an IB radius of about $2 \mathrm{~m}$ and a density difference between the LS inside and outside the balloon less than $0.1 \%$, we estimate a maximum stress of $4 \mathrm{MPa}$ to the balloon film. Considering the deformation of the balloon shape, we set a design requirement of $40 \mathrm{MPa}$ strength, allowing a safety factor of 10 . The breaking strength of our film was measured with a force gauge (ZTA-500N, 
Imada Company) to be about $180 \mathrm{MPa}$ for the direction of film extrusion and about $320 \mathrm{MPa}$ in the perpendicular direction. The strength of welded film is described in section 5.5.

\subsection{Tightness to xenon}

We checked the tightness to xenon of the nylon film using a separated stainless-steel box as shown in figure 5. We filled one side of the box with Xe-LS and the other side with KamLAND LS. We allowed several months to test the penetration of xenon from the Xe-LS to the KamLAND LS through the nylon film. The level of xenon in the KamLAND LS was then measured by gas chromatography (GC-4000 Plus, GL Sciences Company). We did not measure any xenon in this sample of KamLAND LS. The estimated upper limit on the xenon leak rate from the Xe-LS through the IB film is $1.9 \mathrm{~kg}$ over 5 years. This result is an acceptable level for the xenon loss and its impact is negligible in the systematic error for $0 v \beta \beta$ decay search analysis.

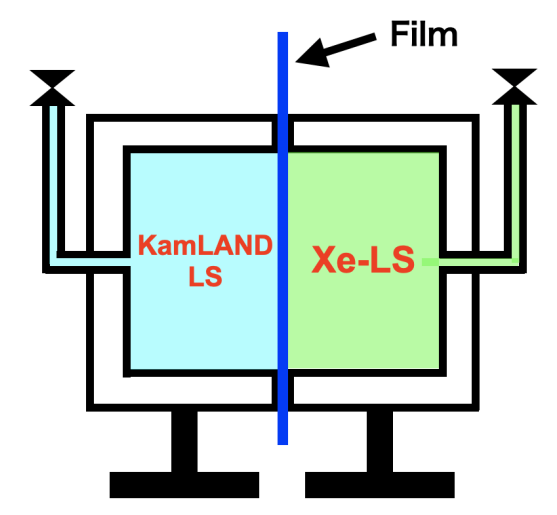

Figure 5. Schematic view of xenon tightness measurement.

\section{Inner balloon fabrication}

\subsection{Clean-room}

The IB was fabricated in Class 1 (ISO 14644-1) clean-rooms (super clean-room, SCR) at the Micro System Integration Center at Tohoku University in Sendai, Japan, about $600 \mathrm{~km}$ from the Kamioka detector site. Outside air is passed through multiple HEPA filters into the building. The entire ceiling area of each SCR is comprised of ULPA filters to maintain a Class 1 environment where the clean air is flowing down from the ceiling to the grating floor. The size of each room is $3.8 \mathrm{~m}$ in height, $14.0 \mathrm{~m}$ in-depth, and $3.6 \mathrm{~m}$ in width. We used two SCRs, a preparation room and a fabrication room for the IB. To avoid the contamination of the fabrication room, the preparation room was used for multiple purposes including welding tests, operational checks of devices, tool cleaning, and changing from normal clean-room suits to SCR suits. The ceiling, walls, floor, and all inner surfaces in the SCR were wiped with ultra-pure water and clean wiping cloths before using the room to avoid particles originating from these surfaces to accumulate on the IB film by static electricity. We used SFIDA and MAX-3003 (MCC Company) wiping cloths in the SCRs. Both cloths were packed at Class-1000 clean-room (FED-STD-209, consistent with Class 5 in ISO 
14644-1), so we washed each cloth with ultra-pure water multiple times (3-5 times). We analyzed the particle flow from human workers, wiping cloths, and other sources and monitored the air flow routes on the work table using particle visualization systems with a green laser. Based on these analyses, we established a procedure requiring that all materials, including the cloths, had to be washed with ultra-pure water, and that SCR wear had to be sent for cleaning after each use.

The custom-made nylon film we used for the IB reaches a static electric potential of about $+3 \mathrm{kV}$ because it was fabricated without filler. It is therefore susceptible to contamination by dust attraction and accumulation. In general, humidity levels above $65 \%$ prevent static electricity, but the SCR humidity is normally less than $65 \%$ between autumn and spring in Japan. We therefore set up a mist generation system just before the ULPA filter sending clean air to the SCR. The AirAKI ${ }^{\circledR}$ (H.IKEUCHI Company) uses ultra-pure water and compressed air to generate 10- $\mu \mathrm{m}$ water mist at up to $144 \mathrm{~L} /$ hour to maintain $65 \%$ humidity in the room. We also introduced two devices to avoid static electricity. A Keyence SJ-F5500 neutralization system was used on all films while handling the film. Another Keyence SJ-H neutralization bar was used when we took nylon films from the roll.

\subsection{Tool cleaning}

We washed all the tools, devices, and other materials used in the SCR by detergent and ultra-pure water, ethanol, isopropyl alcohol, or ethanol with ultra-sonic cleaning. The ethanol was mainly used to remove machine oil from the metal tools. Isopropyl alcohol was used to clean the metal tools that touched the IB and to remove the guide lines marked with pen on the nylon film. The detergent used was Magiclean (Kao Company); this was employed on almost all tools and devices as shown in figure 6. An average of one to two tonnes of ultra-pure water were used per day during the washing.

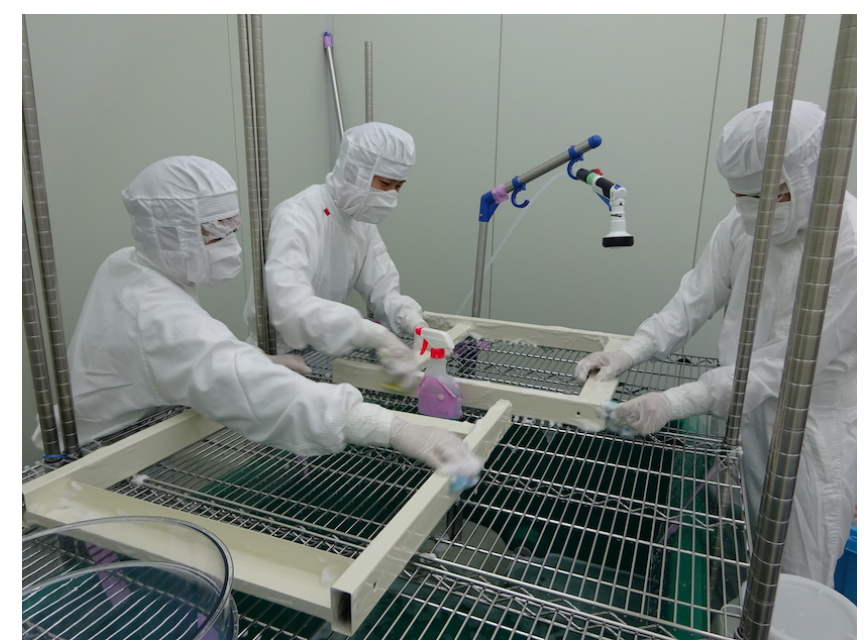

Figure 6. Washing with detergent and ultra-pure water in the preparation room.

\subsection{Corrugated-tube cleaning}

The surface of the corrugated tube, which presents grooves at regular intervals of around $1 \mathrm{~cm}$, was contaminated by large and small particles and by stains of machine and other oils. The outside of the tube was cleaned with a brush and detergent, by wiping with ethanol using a cloth, and by 
ultra-sonic cleaning with ultra-pure water. The inside was cleaned with a brush and detergent, by flushing it with ethanol, and by ultra-sonic cleaning with ultra-pure water. An abridged version of this cleaning procedure had already been applied to the same corrugated tube for the preparation of the KamLAND-Zen 400 experiment: this was shown to yield sufficient cleanliness following in-situ measurements in the KamLAND detector.

\subsection{Film washing, cover setting and cutting}

The first step of IB fabrication was cleaning the nylon film to remove surface contamination. We used ultra-sonic cleaning in ultra-pure water in a stainless-steel tub as shown in figure 7. The film speed was set to about $40 \mathrm{~m} /$ hour, a balance between the need to drain the water off the nylon by gravity and minimizing the risk of water sticking to the film due to surface tension. Light particles were removed from the water by pumping the water at the surface level. Heavier particles were removed through three 8-mm diameter draining holes at the bottom of the cleaning tub. We applied this cleaning procedure twice, thus reducing the ${ }^{238} \mathrm{U}$ level from $9 \times 10^{-12} \mathrm{~g} / \mathrm{g}$ to $2 \times 10^{-12} \mathrm{~g} / \mathrm{g}$, as measured by ICP-MS at NTT-AT Co.
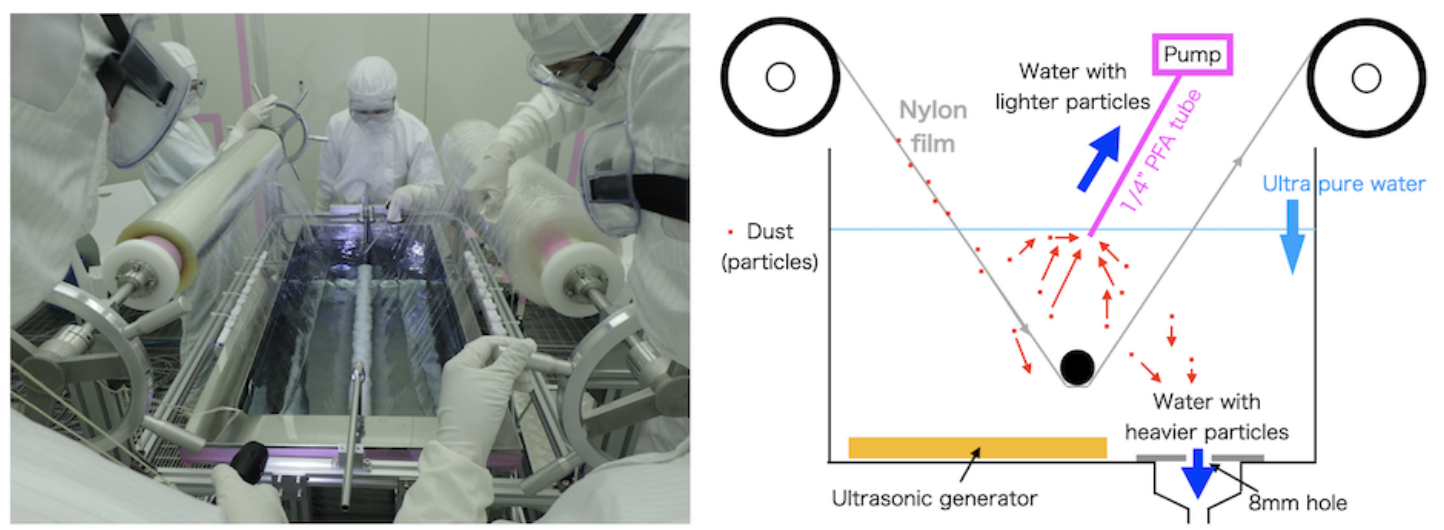

Figure 7. Ultra-sonic film cleaning in ultra-pure water. The film speed was controlled with two rudders by hands. The white center bar is used to submerge the film. Dust in the water was monitored with LED light and removed with a pump. A neutralization bar was set under the film roll to prevent the film from sticking due to static electricity. The ultra-pure water was fed continuously during film washing, and particle contaminant was removed by a pump at the water's surface and by a draining line at the bottom of the tub.

We set cover films over both sides of the IB film during the several-month period required of balloon fabrication to avoid dust accumulation due to static electricity. The cover films were made of the same clean film as the IB and adhered to the IB film by static electricity. When we cut the film into the balloon patterns we cut all three layers simultaneously. During the welding, we turned back the edges of the cover films, and after the balloon sections had been joined, the cover-film edges were unfolded to cover and protect the welding lines.

Nylon film was cut into 32 rectangles with dimensions $7.5 \mathrm{~m} \times 1 \mathrm{~m}$ to make the 24 gore sections, 6 cone sections, the polar cap and polar harness, and one straight tube section, as shown in figure 2. After cutting we made alignment marks on the film for welding position checks. These marks were made with a Microperm 03 pen (Sakura Color Products Company). The ${ }^{238} \mathrm{U}$ level 
of the ink was measured by ICP-MS at NTT-AT Co. to be $1.2 \times 10^{-10} \mathrm{~g} / \mathrm{g}$, an acceptable value considering the tiny amount of remnant ink on the IB.

\subsection{Welding}

Nylon films are typically joined with glue. The Borexino experiment [19] used resorcinol to glue sections of $125-\mu \mathrm{m}$ nylon film together. However, the glues we tested made many holes in $25-\mu \mathrm{m}$ nylon film, and we could not find suitable gluing procedures to avoid this problem. Working with DAIZO Corporation, we instead developed a method to join nylon films by heat welding. The melting point of Nylon- 6 is about $225^{\circ} \mathrm{C}$. The components of our film are $30 \%$ crystallized nylon and $70 \%$ amorphous nylon [20, 21]. As a result, low-temperature welding at less than $225^{\circ} \mathrm{C}$ is possible by molecular motion. However, in our welding tests we found that high-temperature welds were stronger than lowtemperature welds. We therefore tuned the welding parameters based on high-temperature welding.

The primary welding machine was made by Fuji impulse Company (figure 8(a)). It generated impulse heating over a $5 \mathrm{~mm} \times 310 \mathrm{~mm}$ area and could apply heat between 180 to $250{ }^{\circ} \mathrm{C}$ for 0.5 to 10 seconds. NITOFLON ${ }^{\circledR}$ tape (Polytetrafluoroethylene base tape, Nitto Denko Corporation) was applied over the heater surfaces to prevent the melted film from sticking to the device. We found that a two-layer welding with the primary welding machine produced many holes in the weld. To avoid this problem, we added two additional layers of film along the seams, for a total of four layers with a combined thickness of $100 \mu \mathrm{m}$. A schematic of the final welding configuration is shown in figure 8(e). The additional films melt under heating and filled in holes in the IB film. They also avoided overheating the IB film. After testing a range of parameters, we established a welding procedure using $225{ }^{\circ} \mathrm{C}$ heating for 3.5 seconds. The pressing pressure was released when the temperature reached $80^{\circ} \mathrm{C}$. The strength of the welded seams was measured with a force gauge as shown in figure 9(a). Figure 9(b) shows the film strength after welding. This figure indicates that heating through the added films produces a stronger weld than heating the IB film directly. During balloon production, all seams were welded by applying heat through the added films.

Some of the welds required to form the IB presented special challenges. To make the final weld between the gores forming the main body of the sphere, the upper heater of the welder had to be outside the volume of the balloon while the heat-receiving bar had to be inside, a topological impossibility with the primary welding machine. In several regions where weld lines converged we needed shorter welds than the $31-\mathrm{cm}$ lengths of the primary welding machine, namely in the areas near the bottom pole, at the junction between the gores and the upper cone, and at the connection between the upper cone at the straight tube. We worked with Creative Design and Engineering (Laporte, Colorado, U.S.A.) to develop a secondary welding machine (figure 8(b)) to address these issues. The secondary machine features a $10-\mathrm{cm}$ heater and a lower heat-receiving bar designed to allow the second layer of film to pass under the bar. The best welding parameters for the secondary machine were welding at $\sim 225^{\circ} \mathrm{C}$ for 10.0 seconds. Welds made with the secondary machine had strength greater than $160 \mathrm{MPa}$, slightly stronger than the primary machine.

The welding parameters of the primary and secondary machines were tuned to provide the nominal strength. However, welding still periodically resulted in holes along the weld lines. The number of holes in the welds was variable and depended on additional factors that we could not completely control, including the room temperature, the humidity, and the condition of the 

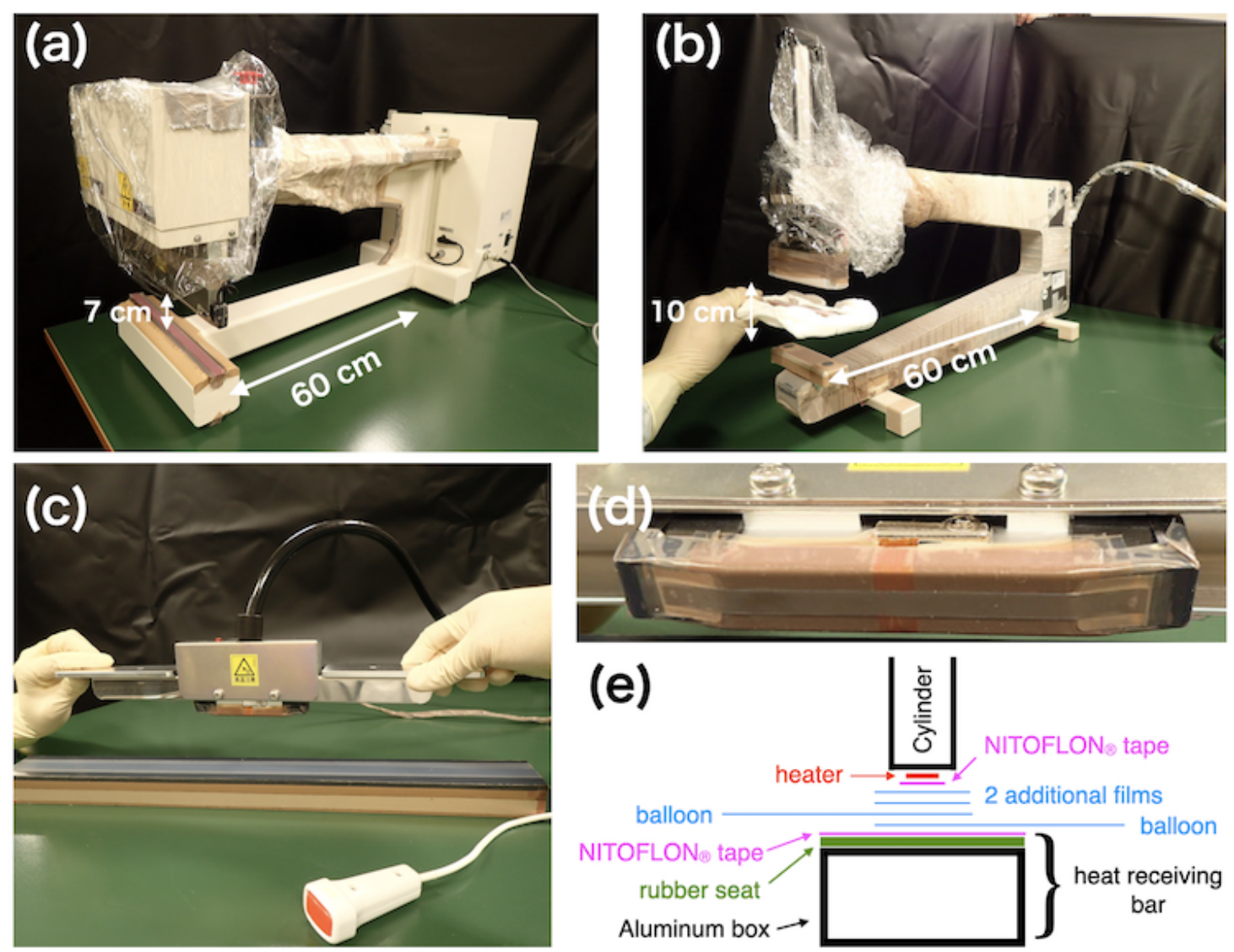

Figure 8. (a) Primary welding machine. (b) Secondary welding machine. The lower heat-receiving bar can be separated and set on top of a different section of the IB in order to weld the final seams. (c) Hand welding machine. (d) The tape-covered heater of the hand welding machine. (e) Schematic of film welding. The four film layers are compressed by the cylinder and heat applied. Layers of NITOFLON ${ }^{\circledR}$ tape under the heater and above the lower rubber seat prevent melted film sticking to the welding device. The heat-receiving bar is used to reflect heat from the heater.

NITOFLON ${ }^{\circledR}$ tape between the IB film and the heater. We therefore developed a methods to patch these holes, as is described in section 5.7.

Most of the welding lines were made in $30-\mathrm{cm}$ increments using the primary welding machine. However, since the heat-receiving bar opposite the heating element in this machine could not be detached, the final weld line in the main body of the sphere was kept open, as were other welds in areas where weld lines converged. These final welds were made in smaller increments with the secondary welding machine (figure $8(\mathrm{~b})$ ), with a few particularly difficult areas done using a hand welding machine (figure 8(c)). Both these smaller machines allow the heat-receiving bar to be positioned inside the balloon volume.

\subsection{Connection cylinder}

The IB and the upper corrugated tube are connected by a plastic cylinder made of PEEK, by using PEEK film holders, additional nylon film, and glue, as shown in figure 3(b). The outer circumference of the connection cylinder and the inner surface of the top of the straight-tube section of the IB 
(a)

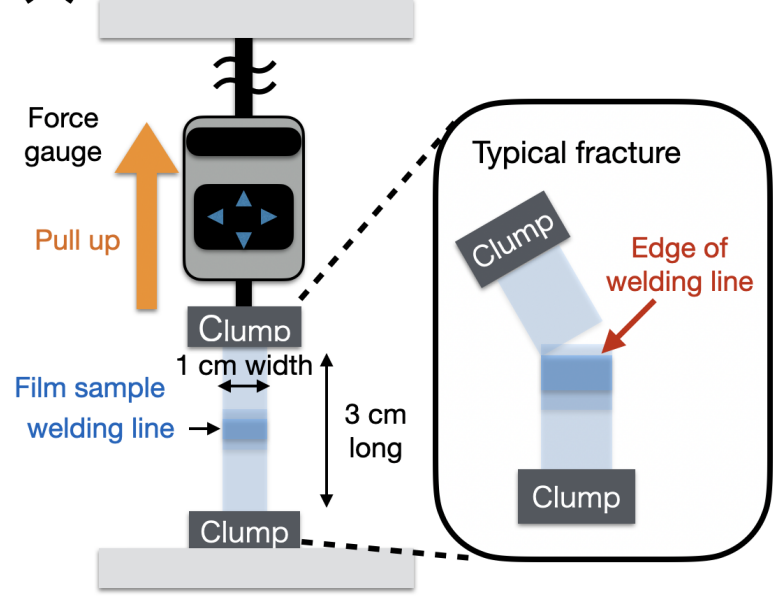

(b)

A case of heating from the 2 additional film side

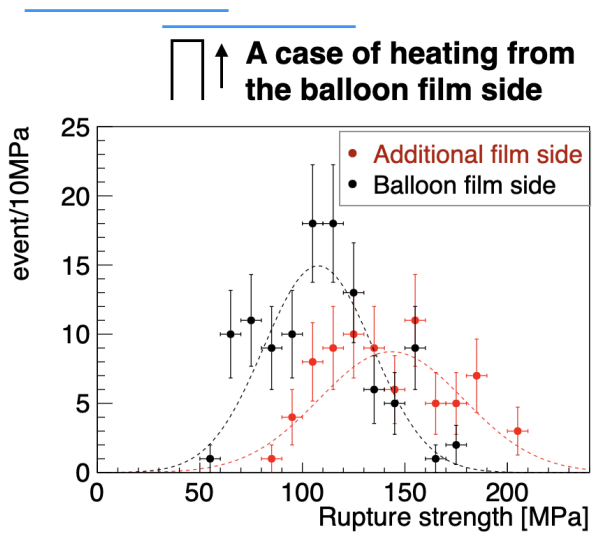

Figure 9. (a) Schematic view of the weld strength test. Fractures typically occur at the edge of the weld. (b) Film strength after welding. The data in red are from welds made with heat applied through the two additional layers of film, while black shows data for welds made with heat applied directly to the IB material.

are a tight fit. Once connected, they can support $20 \mathrm{~kg}$. However, to reduce the risk of slippage of the IB, we clamped the IB film with outer film holders made of PEEK. We applied glue to fill any small gaps that might allow gas or liquid to leak. The glue we used was "Adcoat" a mixture of 91\% AD-76P-1 with 9\% CAT-10L (Toyo-Morton Company, ${ }^{238} \mathrm{U}<1 \times 10^{-11} \mathrm{~g} / \mathrm{g}$ measured with ICP-MS). This glue was selected for its suppleness based on our prior experience with its use as the laminate glue in the construction of the original (outer) KamLAND balloon. It takes more than one day to harden, but it is suitable for these types of connections, which require multiple steps for the overall gluing operation, namely fitting the film onto the cylinder, arranging the glue and additional film layers, and installing the film holders, making a glue with a setting time preferable. We actually allowed 3 days for the glue to harden at room temperature.

\subsection{Leak check and repair}

We checked the balloon for leak points by filling it with helium and using helium leak-check detectors (M-222LD-D, Canon Anelva Corporation, and Adixen, Pfeiffer Vacuum GmbH), as shown in figure 10(a). To avoid introducing dust to the IB surface we covered the helium probes with SFIDA cloth (figure 10(b)). We found two types of leaks, termed z-leaks and y-leaks, as illustrated by figure $10(\mathrm{c})$. The z-leaks appear to be caused by factors including the boiling of water contained in the film, local heat concentration by the NITOFLON ${ }^{\circledR}$ tape adhering to the film, the condition of the heating element, creases in the film, and air remaining between the films. The y-leaks appear to be caused by insufficient welding heat, and also by film creases and local heat concentration. The total number of leak points was about 1,000. Most of these holes were not visible, but they included about 10 holes of about $0.1 \mathrm{~mm}$ width and several y-leaks of about $10 \mathrm{~mm}$ width.

Two methods were used to repair the leak holes made during film welding, both based on the use of additional nylon film, i.e. re-welding and gluing. The largest leak points, mainly torn parts along welding lines, were repaired with re-welding. However, after repeated re-welds the 

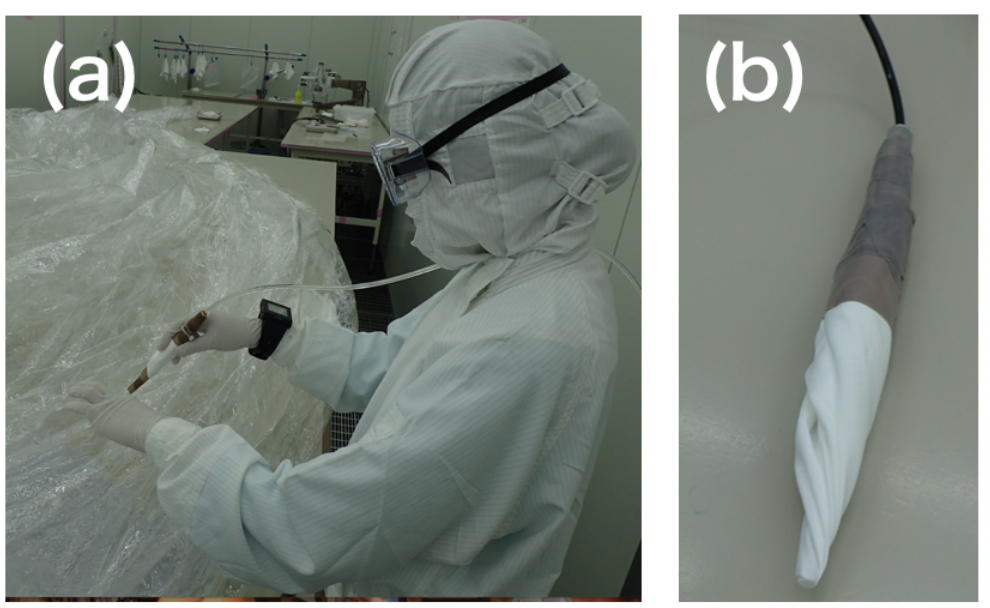

(c)
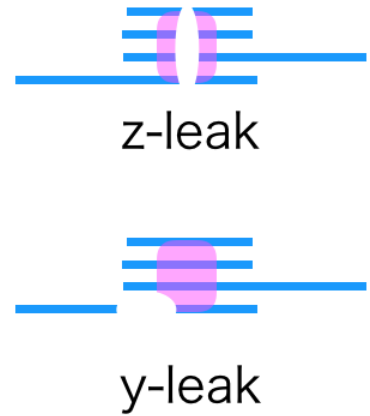

Figure 10. (a) Leak check. The IB was filled with helium and leaks were investigated with a helium detector outside the balloon. The leak rate was viewed via a wrist monitor connected to a camera in front of the helium detector. (b) Close-up photo of leak detector probe covered with SFIDA to avoid the damage to the film or contamination with dust. (c) Definition of welding line leak types. The layers of nylon film are shown in blue, while the pink area represents the hardened nylon melted by the welding processes. Defining the $\mathrm{x}$-axis to lie along the weld line, a z-leak penetrates the weld in the direction normal to the film surface while a y-leak corresponds to a penetration escaping the side of the weld, perpendicular to both a $\mathrm{z}$-leak and the weld line.

nylon film becomes harder, and re-welds create weak points susceptible to damage when the film is folded. Therefore most leaks were repaired with glue and small pieces of nylon film $(10 \mathrm{~mm} \times 10$ $30 \mathrm{~mm}$ ). The glue used was Aron Alpha 202 (Toagosei Company, Ltd.). This glue is a single-liquid adhesive that cures at room temperature and humidity, and has high viscosity. It did not adhere to hardened glue, so it could not be used as the primary film connection method, but its properties were suitable for repairing the many small leaks. The glue passed compatibility tests in LS, and ICP-MS measurements at NTT-AT Co. gave an upper limit on the ${ }^{238} \mathrm{U}$ concentration of $<5 \times 10^{-12} \mathrm{~g} / \mathrm{g}$. The surface of the glue hardened in several seconds, but we allowed more than 12 hours for the full area to solidify. The repaired points were checked with the helium leak detector after the glue hardened.

\subsection{Hanging belts for the inner balloon}

The bottom of the IB rests on the polar harness, which is connected to the 12 load-bearing nylon hanging belts as shown in figure 2. Figure 11(a) shows a schematic view of the polar area including the belt connections. The polar harness, which has a vent hole at the center, was made from a single layer of nylon film. The purpose of the vent hole was to avoid air getting trapped between the harness and the polar cap during installation, which would lead to light reflection that could bias vertex reconstruction. The belts were made of 2 layers of nylon film welded along the center-line and at their ends. To avoid damage from local tensions, the hanging belts were not directly welded to the polar harness film. The position of the vertical belt is constrained by guide tubes that are also made of nylon film and are glued to the polar harness with Aron Alpha 202. The ends of the 12 belts were welded into loops and connected with 2 Vectran strings (figure 11(b)). We also connected horizontal belts to the vertical belts near the polar harness with Aron Alpha 202 to constrain the 
relative position of the belts. The horizontal belts are similar in structure to the vertical belts but their ends were not closed by welding. The position of the belt and harness assembly relative to the polar cap was left unconstrained, as full-size IB installation tests performed for the KamLAND-Zen 400 balloon demonstrated successful positioning of the polar harness directly below the polar cap in four out of four attempts. The belt and harness assembly was set to the IB just before packing and delivery to the detector site.
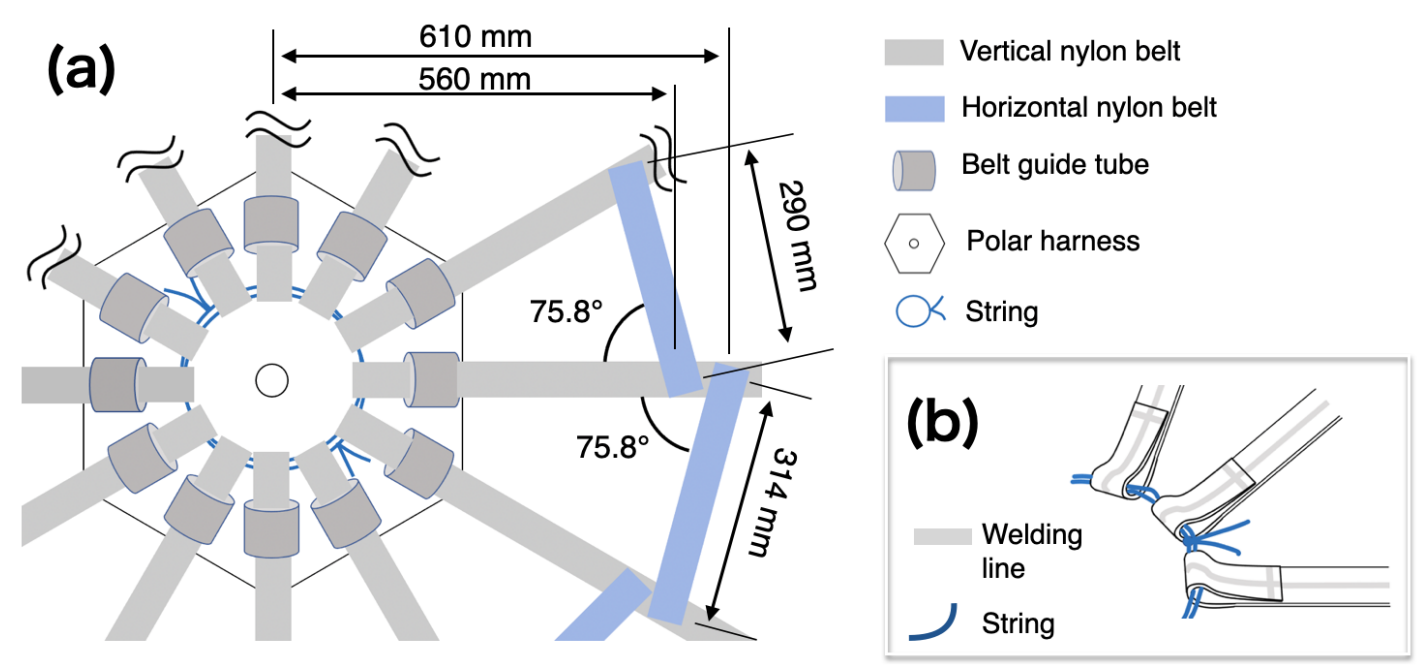

Figure 11. (a) Schematic view of the polar area detailing the hanging belts for the IB. The connection angle of the two belts is designed to be parallel to the equator when the balloon is inflated. (b) Schematic view of the connections between the ends of the 12 vertical belts with the 2 Vectran strings.

\subsection{Packing}

The interior of KamLAND can only be accessed via a narrow opening through the top "chimney" region of the detector (see figure 1). To pass through this space we folded the IB to within a 50-cm diameter. To protect it from damage and to avoid flexing, especially in the small clean-room above the chimney, the IB was folded and packed inside commercial nylon and PTFE sheets as shown in figure 12. The PTFE sheets were closed by 1/8" PFA (perfluoroalkoxy alkanes) tubes, released by pulling out the tubes. The strings tied to PTFE and nylon sheets were used to pull up sheets from the detector after sinking the IB (section 6.5). A custom plastic delivery box was made by the Naigai TEC Corporation. The inner surface was lined with film made by Daizo Corporation (KS137-205). KS137-205 is a 205- $\mu \mathrm{m}$ film made of aluminum vapor deposition EVOH with low density polyethylene on both surfaces for gas tightness. After packing the IB inside, the box was set inside a $1.8 \mathrm{~m} \times 1.8 \mathrm{~m} \times 0.7 \mathrm{~m}$ bag made of KS137-205, and it was sealed via welding. This airtight bag kept the balloon in the SCR air and humidity. 


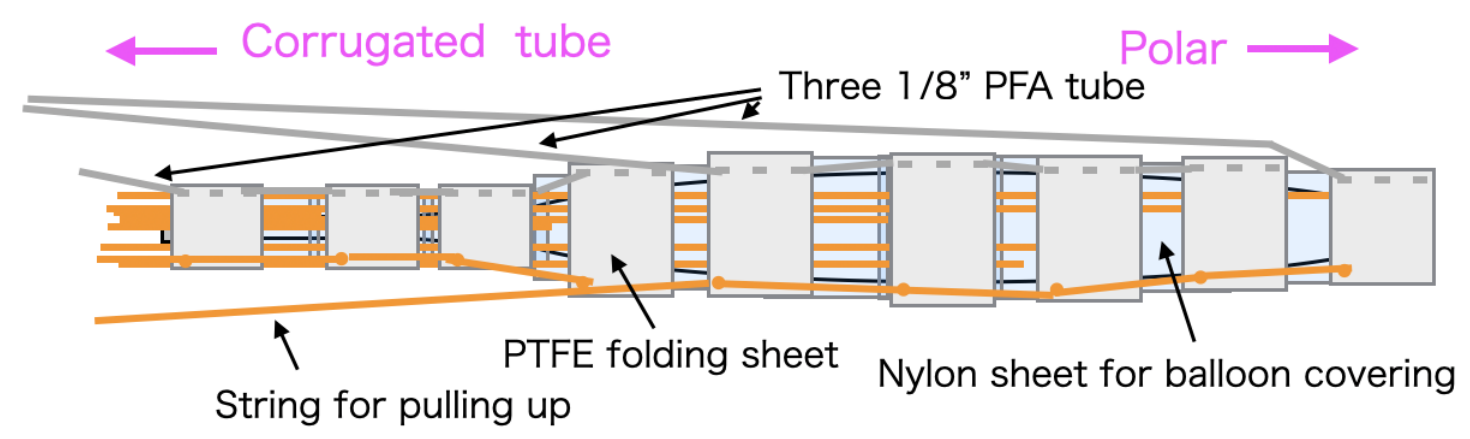

Figure 12. Schematic view of how the IB was folded and packed. The folded balloon was covered with a nylon sheet, held in place by sheets of PTFE. The PTFE sheets were closed by $1 / 8$ " PFA tubes, released by pulling out the tubes. The strings attached to the PTFE and nylon cover sheets allowed them to be extracted from KamLAND following the IB insertion.

\section{Installation in the detector}

\subsection{Onsite preparation}

In order to insert the IB inside the $\mathrm{OB}$, a clean tent was built on the top of the KamLAND detector. The clean-room class grade was between 10-100 (FED-STD-209) depending on the area and the number of people in the tent. The inner surface of the room was cleaned with lint rollers and by wiping with ultra-pure water. The ultra-pure water, with electrical resistivity greater than $18.2 \mathrm{M} \Omega \cdot \mathrm{cm}$, was made using reverse osmosis apparatus with a RFU554CA (Advantec MFS, Inc.) filter. It was used for wiping down surfaces, washing gloved hands, and keeping tools clean. The tables, tools, wipers, and other materials were all delivered to the KamLAND site from Sendai after being cleaned and packed in the SCR.

\subsection{Corrugated tube connection}

A specific procedure was developed and set up in order to assemble the corrugated tube. The individual steps consisted in:

1. Set two O-rings in adjacent "valley" near the end of the corrugated tube (see figure 3).

2. Insert the corrugated tube into the PEEK connection cylinder.

3. Leak check the connection.

4. Apply Adcoat glue at the edge of the PEEK cylinder.

5. Wait 5 days for the adhesive to harden.

6. Connect the grip piece.

We used two O-rings for each connection because we found that the deformation of the corrugated tube led to leaks when only one was used. We added glue to ensure leak tightness as the O-rings alone may leak (Xe-)LS or gases. 


\subsection{String guide}

The string guide (figure 2) drives the 12 strings, preventing them from touching the outer balloon or the chimney of the detector, and avoiding any entanglement of the strings themselves. The string guide is positioned under a light-blocking black film at the bottom of the chimney. We located it as high as possible to prevent the nylon support belts from cutting into the IB. The string guide is connected to the corrugated tube. It can be rotated relatively to the fixed corrugated tube to remove any twisting of the balloon or belts and ropes caused by the installation processes.

\subsection{Monitoring camera}

We cannot view the interior of the detector directly through the upper opening because the hole area at the bottom of chimney is covered by black sheets to block light from the outside. We used two monitoring cameras compatible with LS to oversee the IB installation and to ensure the safety of both the IB and the rest of the detector. After the installation, the cameras were used to regularly monitor the condition of the detector and IB for several months. The primary camera, shown in figure 13(a), is a high-resolution Internet Protocol (IP) camera that can resolve small details and view the whole IB and detector. This camera was covered by a stainless-steel housing and a UV-transparent acrylic dome for use in LS. The power supply and data transfer are through a Power over Ethernet (PoE) LAN cable encased in PFA tubing. The primary monitoring camera was installed before the IB installation and was used to monitor the IB during installation, to monitor balloon expansion during LS filling, to search for signs of leakage, and to assist the positioning and retracting of the filling/draining tube. Figure 13(b) shows the secondary camera, based on a camera designed for pipe inspection (Wöhler VIS 350, Wöhler Technik GmbH). This camera was used to check the vicinity of the IB. The polycarbonate cover dome of this camera was replaced with UV-transparent acrylic for use in the LS. The fiberglass cable was also replaced with one with a fluorine surface compatible with the LS. We also positioned LED lights in the detector, illuminated only while actively monitoring the IB (figure 13(c)).
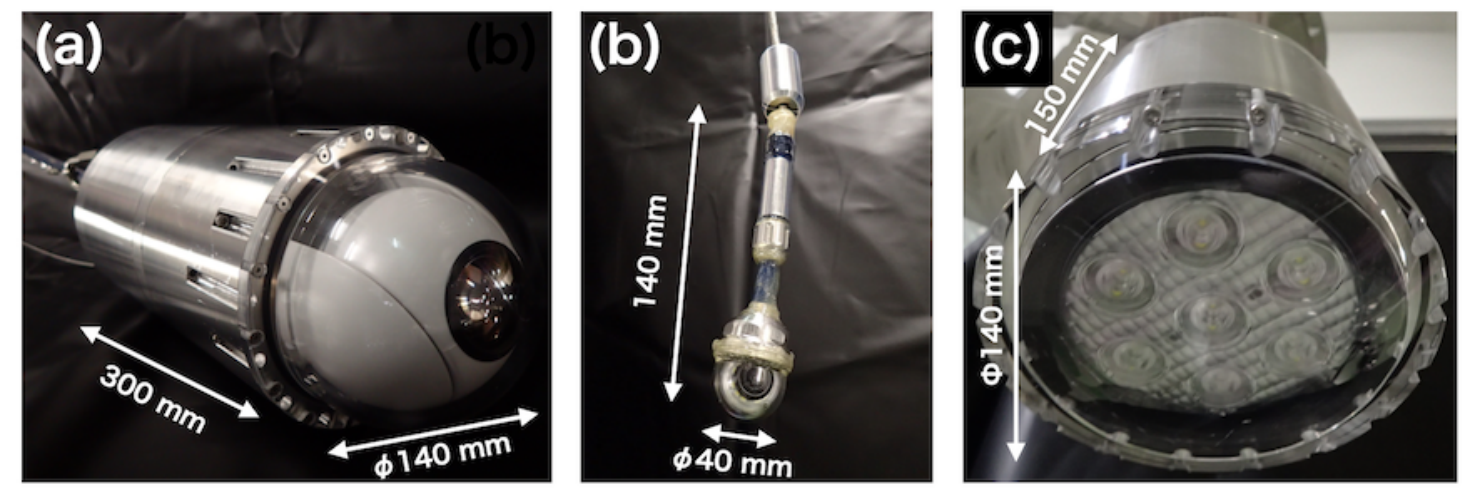

Figure 13. (a) Primary monitor camera. The IP camera is encased in a stainless steel and acrylic cover for use in the LS. Power and data are both transferred via a PoE cable encased in PFA that runs to the top of the detector. (b) Secondary camera. The camera cover and cable were replaced by acrylic and a fluorine-surface cable, respectively. (c) Lighting for detector interior. LED lights $(2,200 \mathrm{~lm})$ with encasement similar to that of the primary camera. 


\subsection{Installation}

The densities of nylon and PTFE are $1.2 \mathrm{~g} / \mathrm{cm}^{3}$ and $2.2 \mathrm{~g} / \mathrm{cm}^{3}$, respectively, while that of the KamLAND LS is $0.77728 \pm 0.00004 \mathrm{~g} / \mathrm{cm}^{3}$. Therefore, the IB with its folding covers would ideally sink into the LS. However, due to air remaining in the IB, lowering it into the detector is actually not so easy. We introduced pseudocumene-rich LS with a density of about $0.78 \mathrm{~g} / \mathrm{cm}^{3}$ into the IB to extract the residual air and to decrease the balloon's buoyancy. As we lowered the IB, we repeated the step-by-step filling in 5-10 L increments. We lowered the balloon as shown in figure 14, keeping the balloon folded in its nylon and PTFE covers (figure 12). Once this was in position, we removed the 1/8" PFA tubes that secured the PTFE cover sheets so that the sheets would detach from the balloon. We then used the strings attached to the nylon and PTFE covers to pull them up to the access area at the top of the detector and remove them.
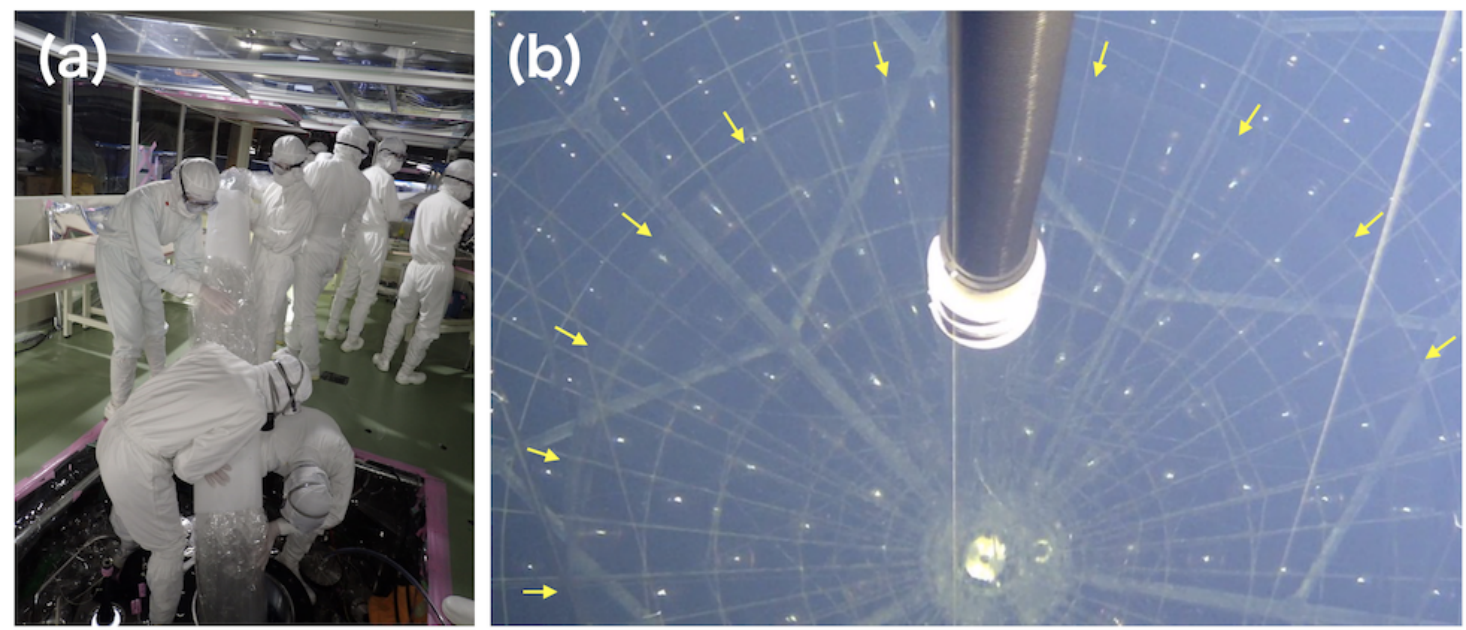

Figure 14. (a) Installation of the IB into the detector. (b) Photograph of the IB installed in the detector, taken with the monitoring camera. It is difficult to see the IB directly; the edge shown by arrows can be located by noting the discontinuities in the imaged stainless steel structures.

\subsection{Inner balloon inflation and liquid scintillator filling}

The IB was inflated by filling it with decane and pseudocumene based LS (non-Xe-loaded LS) with a density $0.77732 \mathrm{~g} / \mathrm{cm}^{3}$. Since the density of LS increases by $0.1 \% /{ }^{\circ} \mathrm{C}$ at temperatures near $10^{\circ} \mathrm{C}$, we maintained the temperature of the added scintillator within the range $9.5-10.5^{\circ} \mathrm{C}$, compared to a temperature at the center of KamLAND of about $12.0^{\circ} \mathrm{C}$. The LS flow rate was about $270 \mathrm{~L} /$ hour, and the total volume of LS filled to expand the IB was $30.5 \mathrm{~m}^{3}$. The effective radius of the IB based on the filled non-Xe-loaded LS is $1.90 \mathrm{~m}$.

\subsection{Filling of xenon loaded liquid scintillator}

The non-Xe-loaded LS was replaced by Xe-LS by circulation after the IB was leak-checked. The leak checked was performed by monitoring the IB weight with load cells, the shape stability was verified with the primary cameras and the decane concentration in the KamLAND-LS was measured using gas chromography. A 3/4" PFA tube was used as a filling line (figure 15(a)), with its end positioned $200 \mathrm{~mm}$ above the bottom of the balloon (figure 15(d)). A similar tube, whose end is 
positioned in the cone area, was used as draining line (figure 15(b)(d)). The xenon was dissolved in the non-Xe-loaded LS removed from the IB by bubbling xenon in a dedicated circulation system (Xe-system, figure 15(c)). The non-Xe-loaded LS was first degassed in the main tank. Both xenon and decane were then added to the LS in the sub-tank to control the density of the Xe-LS. The Xe-LS was sampled from the sub-tank flow line and the xenon concentration was measured by gas chromatography before filling to the IB. The Xe-LS was tuned to have a density of about $0.77741 \mathrm{~g} / \mathrm{cm}^{3}$ and temperature of about $10.5^{\circ} \mathrm{C}$. This Xe-LS had a slightly higher density than the non-Xe-loaded LS in the IB, and this allowed to keep the non-Xe-loaded LS separated from the XeLS in the IB. The Xe-LS volume was monitored by ${ }^{214} \mathrm{Bi}^{-214} \mathrm{Po}$ coincident events of ${ }^{222} \mathrm{Rn}$ daughter nuclei as shown in figure $15(\mathrm{f})$. The source of the ${ }^{222} \mathrm{Rn}$, whose half-life is 3.8 days, is emanation from the inner surfaces of the stainless steel tanks in the Xe-system, and from the piping between the Xe-system and the PFA tubes at the detector. The Xe-LS and non-Xe-loaded LS flow rate was about $150 \mathrm{~L} /$ hour, with about $600 \mathrm{~L}$ in one filling batch due to the size of sub-tank in the first cycle. We performed roughly two full volume exchanges of the Xe-LS to maximize the xenon concentration (figure 15(e)), as some amount of the Xe-LS mixes with the non-Xe-loaded LS at the boundary. We applied continuous Xe-LS circulation between the sub-tank and the IB in the second cycle to maximize the xenon concentration in Xe-LS. The total amount of xenon dissolved into the balloon was measured by the weight of gas storage to be $745 \mathrm{~kg}$, corresponding to $677 \mathrm{~kg}$ of ${ }^{136} \mathrm{Xe}$. This is consistent with the estimate from the Xe-LS xenon concentration analysis by gas chromatography.

\section{Inner balloon contamination}

The levels of ${ }^{238} \mathrm{U}$ and ${ }^{232} \mathrm{Th}$ in and on the deployed IB film were assayed in-situ by measuring the excess spectral strength due to these sources reconstructing at the radial position of the balloon surface. Figure 16 shows the radial distribution of events with visible energy range $2.30 \mathrm{MeV}$ to $2.70 \mathrm{MeV}$, which is dominated by ${ }^{214} \mathrm{Bi}$ decay events. We estimated the number of events of ${ }^{214} \mathrm{Bi}$ and $2 v \beta \beta$ by fitting the observed data simultaneously for both components. The other backgrounds such as muon spallation products, external $\gamma$ from the stainless steel containment vessel, ${ }^{238} \mathrm{U}$ and ${ }^{232} \mathrm{Th}$ series in LS, and solar ${ }^{8} \mathrm{~B}$ neutrinos were estimated using the KamLAND LS volume and fixed in the fitting. From this analysis and that of ${ }^{208} \mathrm{Tl}$, we evaluated ${ }^{238} \mathrm{U}$ and ${ }^{232} \mathrm{Th}$ levels in the IB as $3 \times 10^{-12} \mathrm{~g} / \mathrm{g}$ and $4 \times 10^{-11} \mathrm{~g} / \mathrm{g}$, respectively. Contamination was reduced by a factor of 10 from the KamLAND-Zen 400 phase of our $0 v \beta \beta$ search [7]. The amount of ${ }^{238} \mathrm{U}$ contamination is consistent with pre-installation measurements of the raw nylon film, thus proving that we succeeded in constructing a new IB without polluting the detector.

Looking ahead, to reach even lower background levels due to radioactive contamination of the IB, we would need to either use cleaner nylon film, produced in a clean-room, or to use a different technique like introducing a self-vetoing balloon made from scintillating material [22].

\section{Summary}

We fabricated a balloon to contain the xenon-loaded liquid scintillator for the KamLAND-Zen 800 experiment. The balloon was made of nylon film and assembled in an ultra-clean environment. We successfully installed the balloon and inflated it inside the KamLAND detector by filling it with density tuned liquid scintillator without xenon. Finally, we successfully replaced the liquid 

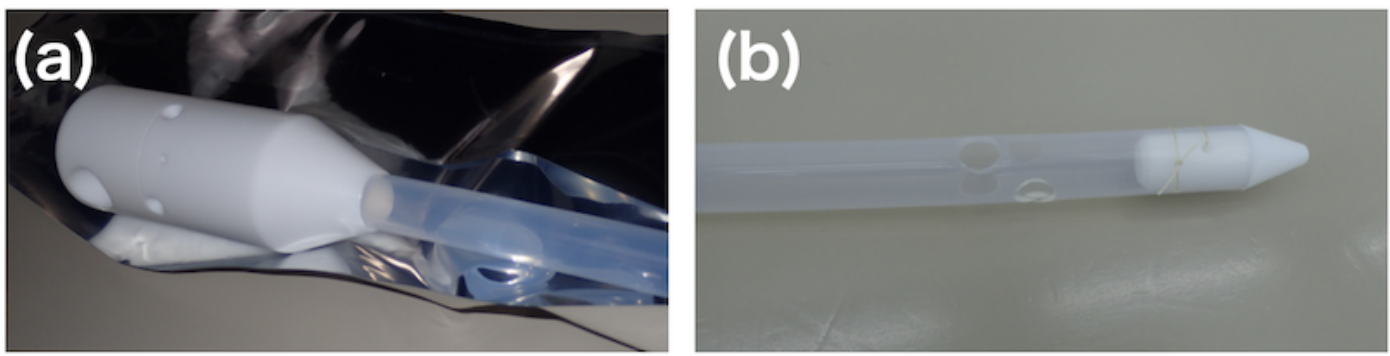

(c)

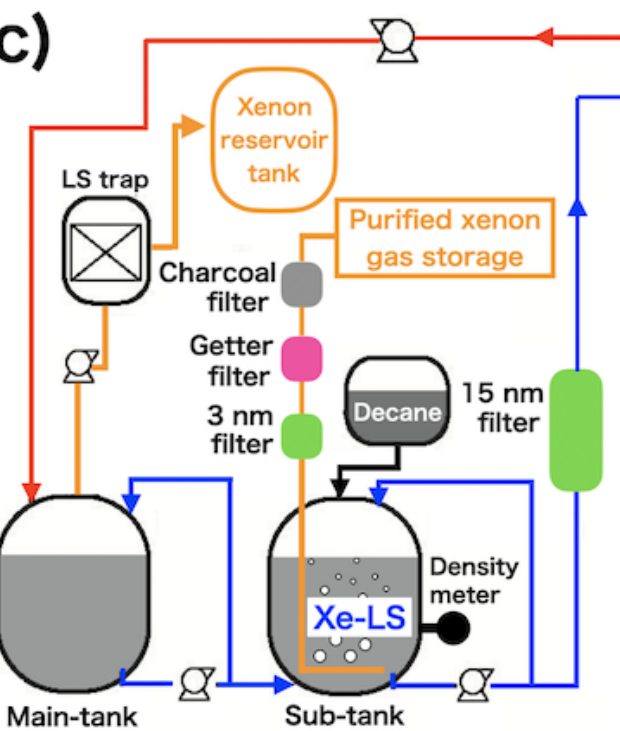

(e)
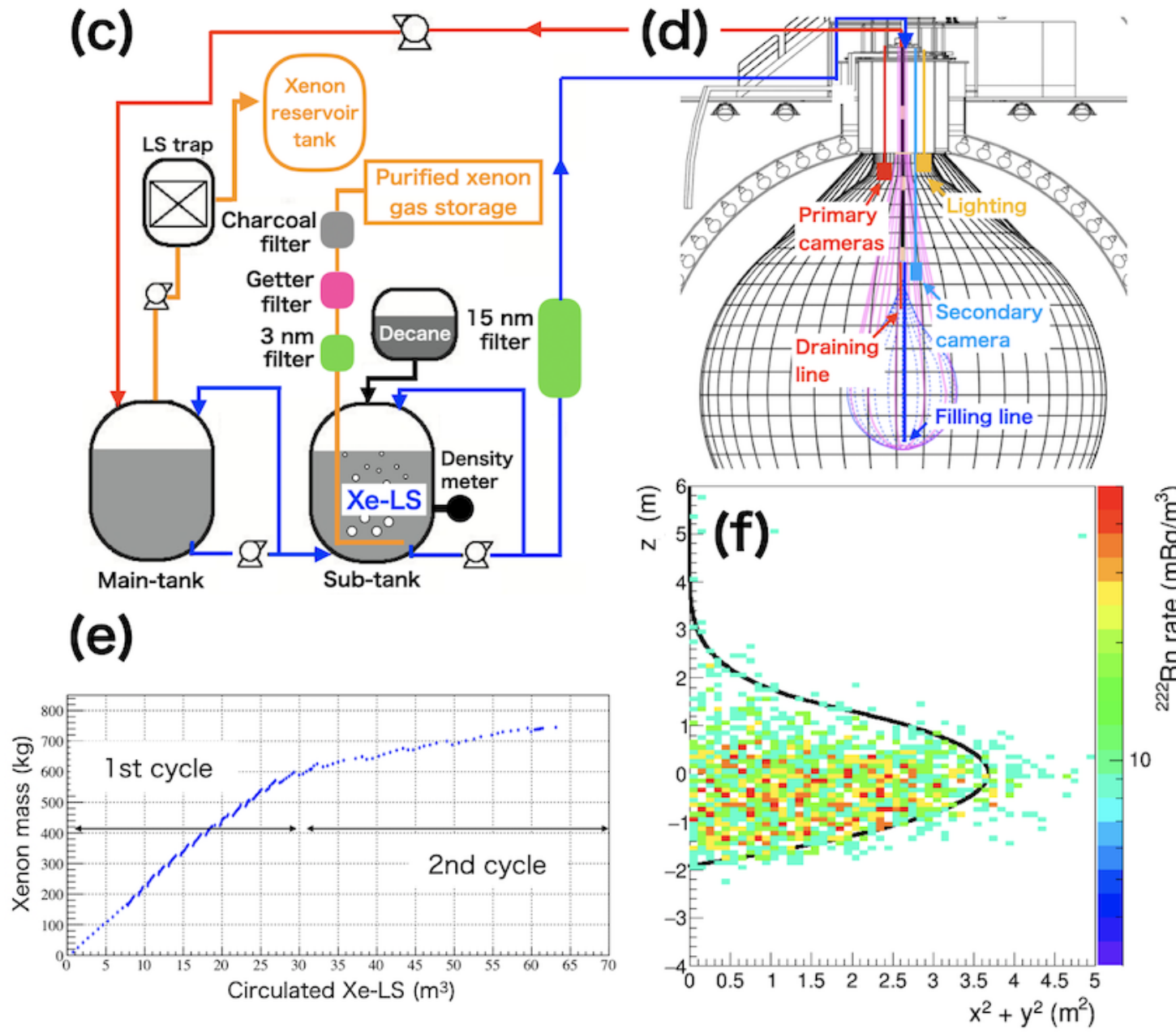

Figure 15. (a) The end of Xe-LS filling line. (b) The end of LS draining line. (c) Diagram of the Xe-loading circulation loop, replacing LS with Xe-LS. (d) The setup positions of cameras and tubes in the detector. Two primary cameras placed diagonally across the corrugated tube monitor the entire balloon. (e) Xenon mass vs. circulated LS volume. (f) The vertex distribution of ${ }^{214}$ Po events during Xe-LS filling. The black line is the expected outline of the IB film position.

scintillator with the xenon-loaded liquid scintillator. The ${ }^{238} \mathrm{U}$ contamination of the balloon is consistent with the level in the initial nylon, indicating that we built the balloon without significant additional contamination. This contamination level shows a factor-10 improvement with respect to KamLAND-Zen 400. 


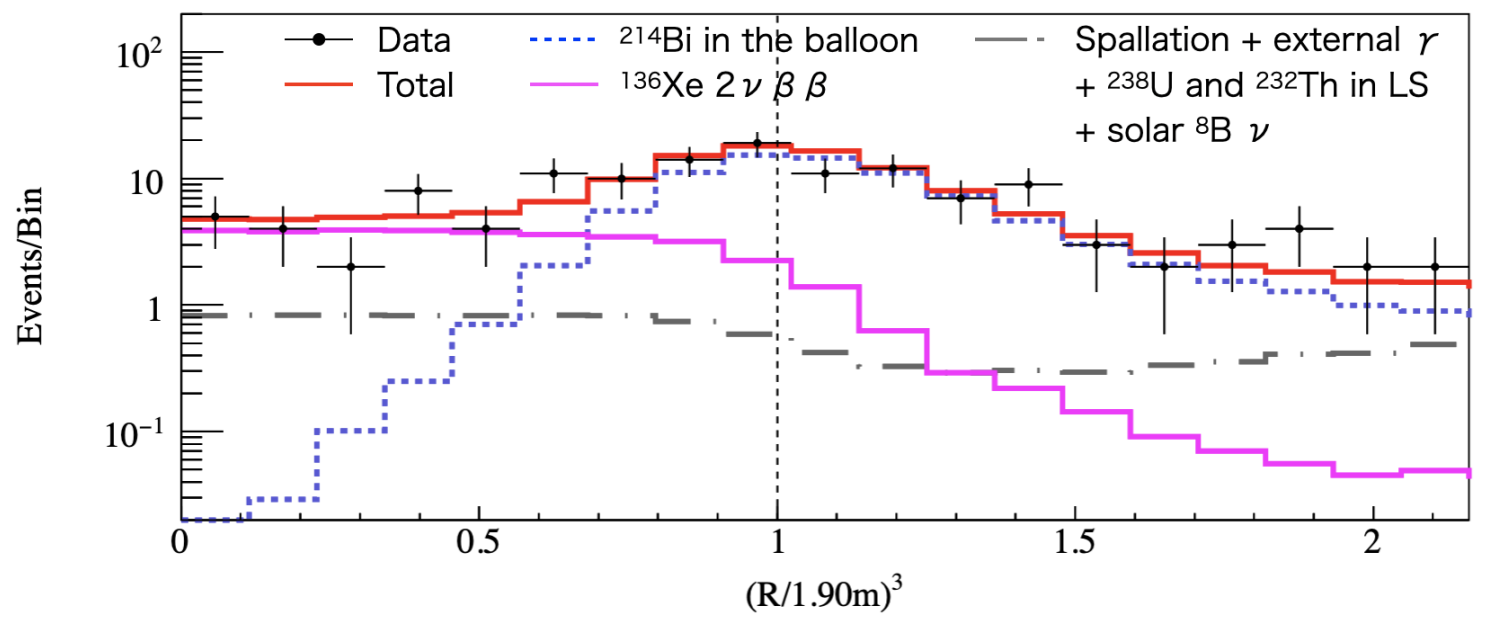

Figure 16. Reconstructed radial distribution of events with visible energy $2.30-2.70 \mathrm{MeV}$ after filling the IB with Xe-LS. The radial position is normalized to the nominal IB radius of $1.90 \mathrm{~m}$. The observed data are plotted in black with error bars. The red line is the total fit to the data, while the blue dotted line is the best-fit contribution from ${ }^{214} \mathrm{Bi}$ in the balloon film, the magenta curve is $2 v \beta \beta$ decay, and the gray dashdotted line represents the sum of all other backgrounds: muon spallation products of carbon and xenon, external $\gamma,{ }^{238} \mathrm{U}$ and ${ }^{232}$ Th-series in LS, and solar ${ }^{8} \mathrm{~B}$ neutrinos.

\section{Acknowledgments}

The authors would like to thank the DAIZO Corporation for developing nylon film welding with us, Toyobo Company for developing and providing the custom-made nylon film, and Creative Design and Engineering for developing secondary welding machine. We are grateful to the institutes and companies described in the main text, all of whom have been critical in the production of the IB and the development of associated technologies. The KamLAND-Zen experiment is supported by JSPS KAKENHI Grants No. 21000001 and No. 26104002; the World Premier International Research Center Initiative (WPI Initiative), MEXT, Japan; Stichting FOM in the Netherlands; and under the U.S. Department of Energy (DOE) Contract No. DE-AC02-05CH11231, as well as other DOE and NSF grants to individual institutions. The Kamioka Mining and Smelting Company has provided service for activities in the mine. We acknowledge the support of NII for SINET4. This work was supported by JSPS KAKENHI Grants No. 21244025, No. 25220704, No. 17H01120, No. 21684008, No. 26287035, and No. 18J10498, NSF Award 1806440 and a UVLAC-Hayashi MISTI Seed Grant. This work was partly supported by the Graduate Program on Physics for the Universe (GP-PU), Tohoku University.

\section{References}

[1] J. Schechter and J.W.F. Valle, Neutrino decay and spontaneous violation of lepton number, Phys. Rev. D 25 (1982) 774.

[2] P. Minkowski, $\mu \rightarrow$ ey at a rate of one out of $10^{9}$ muon decays?, Phys. Lett. B 67 (1977) 421.

[3] T. Yanagida, Horizontal gauge symmetry and masses of neutrinos, Conf. Proc. C 7902131 (1979) 95. 
[4] M. Gell-Mann, P. Ramond and R. Slansky, Complex spinors and unified theories, Conf. Proc. C 790927 (1979) 315 [arXiv: 1306. 4669].

[5] M. Fukugita and T. Yanagida, Baryogenesis without grand unification, Phys. Lett. B 174 (1986) 45.

[6] M. Redshaw, E. Wingfield, J. McDaniel and E.G. Myers, Mass and double-beta-decay $Q$ value of ${ }^{136}$ Xe, Phys. Rev. Lett. 98 (2007) 053003.

[7] KamLAND-Zen collaboration, Search for Majorana neutrinos near the inverted mass hierarchy region with KamLAND-Zen, Phys. Rev. Lett. 117 (2016) 082503 [Addendum ibid. 117 (2016) 109903] [arXiv: 1605.02889].

[8] KAMLAND collaboration, First results from KamLAND: evidence for reactor anti-neutrino disappearance, Phys. Rev. Lett. 90 (2003) 021802 [hep-ex/0212021].

[9] KamLAND collaboration, Reactor on-off antineutrino measurement with KamLAND, Phys. Rev. D 88 (2013) 033001 [arXiv: 1303. 4667].

[10] T. Araki et al., Experimental investigation of geologically produced antineutrinos with KamLAND, Nature 436 (2005) 499.

[11] KamLAND collaboration, Partial radiogenic heat model for earth revealed by geoneutrino measurements, Nature Geo. 4 (2011) 647.

[12] KAmLAND collaboration, Measurement of the ${ }^{8} B$ solar neutrino flux with the KamLAND liquid scintillator detector, Phys. Rev. C 84 (2011) 035804 [arXiv: 1106.0861].

[13] KamLAND collaboration, ${ }^{7}$ Be solar neutrino measurement with KamLAND, Phys. Rev. C 92 (2015) 055808 [arXiv: 1405.6190].

[14] KAMLAND-ZEN collaboration, Measurement of the double- $\beta$ decay half-life of ${ }^{136} X e$ with the KamLAND-Zen experiment, Phys. Rev. C 85 (2012) 045504 [arXiv : 1201.4664].

[15] XMASS collaboration, Distillation of liquid xenon to remove krypton, Astropart. Phys. 31 (2009) 290 [arXiv: 0809.4413].

[16] KamLAND collaboration, Production of radioactive isotopes through cosmic muon spallation in KamLAND, Phys. Rev. C 81 (2010) 025807 [arXiv: 0907.0066].

[17] T. Hachiya et al., Delayed coincidence with a day-scale window for tagging ${ }^{232}$ Th series isotopes in KamLAND, J. Phys. Conf. Ser. 1468 (2020) 012257.

[18] A. Gando, First results of neutrinoless double beta decay search with KamLAND-Zen, Ph.D. thesis, Tohoku University, Sendai, Japan (2012).

[19] J. Benziger et al., The nylon scintillator containment vessels for the borexino solar neutrino experiment, Nucl. Instrum. Meth. A 582 (2007) 509 [physics/0702162].

[20] K. Matsumoto, T. Kuroki and R. Imamura, Molecular orientation of the biaxially stretched nylon-6 films prepared by dry-process, Sen'i Gakkaishi 30 (1974) T494.

[21] K. Ito from Toyobo company, private communication.

[22] S. Obara, Y. Gando and K. Ishidoshiro, Scintillation balloon for neutrinoless double-beta decay search with liquid scintillator detectors, PTEP 2019 (2019) 073H01 [arXiv: 1903. 10736]. 